\title{
Vibrissa-Based Object Localization in Head-Fixed Mice
}

\author{
Daniel H. O'Connor, Nathan G. Clack, Daniel Huber, Takaki Komiyama, Eugene W. Myers, and Karel Svoboda \\ Janelia Farm Research Campus, Howard Hughes Medical Institute, Ashburn, Virginia 20147
}

Linking activity in specific cell types with perception, cognition, and action, requires quantitative behavioral experiments in genetic model systems such as the mouse. In head-fixed primates, the combination of precise stimulus control, monitoring of motor output, and physiological recordings over large numbers of trials are the foundation on which many conceptually rich and quantitative studies have been built. Choice-based, quantitative behavioral paradigms for head-fixed mice have not been described previously. Here, we report a somatosensory absolute object localization task for head-fixed mice. Mice actively used their mystacial vibrissae (whiskers) to sense the location of a vertical pole presented to one side of the head and reported with licking whether the pole was in a target (go) or a distracter (no-go) location. Mice performed hundreds of trials with high performance ( $>90 \%$ correct) and localized to $<0.95 \mathrm{~mm}\left(<6^{\circ}\right.$ of azimuthal angle). Learning occurred over 1-2 weeks and was observed both within and across sessions. Mice could perform object localization with single whiskers. Silencing barrel cortex abolished performance to chance levels. We measured whisker movement and shape for thousands of trials. Mice moved their whiskers in a highly directed, asymmetric manner, focusing on the target location. Translation of the base of the whiskers along the face contributed substantially to whisker movements. Mice tended to maximize contact with the go (rewarded) stimulus while minimizing contact with the no-go stimulus. We conjecture that this may amplify differences in evoked neural activity between trial types.

\section{Introduction}

Genetically encoded molecules facilitate measurement and control of the activity of subsets of neurons (Luo et al., 2008). Expression in specific cell types will link activity in these cells with behavior. These molecular tools are most powerful when applied to genetic model organisms such as the mouse, which allow targeting transgenes to specific cell types (O'Connor et al., 2009). Dissecting the mechanisms underlying behavior will also require tight control over behavior, including sensory stimuli and motor output, ideally with temporal precisions comparable with the shortest timescales of neural computation (1-10 ms). In addition, large numbers of repeated trials are desirable to uncover quantitative relationships between neural activity and behavioral variables. Awake-behaving primates are currently the gold standard for behavioral neurophysiology (Evarts, 1968; Wurtz, 1968). Head fixation is critical in these experiments, providing excellent behavioral control and repeatability across trials. Experiments in head-fixed mice have so far been limited to reflexive behaviors, such as the vestibulo-ocular reflex (Boyden and Raymond, 2003).

We developed a vibrissa-based object localization task for head-fixed mice.

Rats and mice move their large vibrissae (whiskers) through the space surrounding the head, often in a rhythmic pattern, to

\footnotetext{
Received Aug. 3, 2009; revised Dec. 21, 2009; accepted Dec. 24, 2009.

This work was supported by the Howard Hughes Medical Institute. We thank Brenda Shields, Nima Ghitani, and Takashi Sato for help with histology; Jim Cox and Margie Fortman for husbandry; Daniel Flickinger, Tanya Tabachnik, Jason 0sborne, and Steve Bassin for help with mechanical design and machining; Gus Lott for help with videography; Lorenz Pammer for discussions and help with whisker tracking; and Dmitry Rinberg, Takashi Sato, and Matt Smear for comments on this manuscript.

Correspondence should be addressed to Dr. Karel Svoboda, Janelia Farm Research Campus, Howard Hughes Medical Institute, 19700 Helix Drive, Ashburn, VA 20147. E-mail: svobodak@janelia.hhmi.org.

DOI:10.1523/JNEUROSCI.3762-09.2010

Copyright $\odot 2010$ the authors $\quad 0270-6474 / 10 / 301947-21 \$ 15.00 / 0$
}

locate landmarks (Hutson and Masterton, 1986; Knutsen et al., 2006; Mehta et al., 2007) and identify textures (Guić-Robles et al., 1989; Carvell and Simons, 1990; Diamond et al., 2008). Whiskers arise from an array of follicles within the mystacial pad (Dörfl, 1982). Common modes of whisking include "exploratory" whisking (Welker, 1964; Carvell and Simons, 1990; Knutsen et al., 2005; Voigts et al., 2008), comprising bilaterally symmetric, high-amplitude movements (5-15 Hz, rats; $20 \mathrm{~Hz}$, mice), and higher-frequency, lower-amplitude "foveal" whisking (15-25 $\mathrm{Hz}$, rats) (Berg and Kleinfeld, 2003). Rats also exhibit asymmetric whisking (Mitchinson et al., 2007; Towal and Hartmann, 2008) and irregular whisker movements during a horizontal object localization task (Knutsen et al., 2006). Active whisking is not required to solve all spatial tasks: rats can determine the width of an aperture after sectioning of the facial nerve (Krupa et al., 2001). The whisking strategies used by rodents to solve spatial localization problems are incompletely understood. Moreover, the limits of absolute localization (that is, memory-guided localization in laboratory coordinates, rather than relative positions) are unknown.

Here we trained head-fixed mice to locate objects in the posteroanterior (horizontal) direction, similar to previous tasks in freely moving (Knutsen et al., 2006) and partially restrained (Mehta et al., 2007) rats. Mice learned the task after 1-2 weeks of training and performed hundreds of trials per session. Whiskers and the vibrissa area of the primary somatosensory (barrel) cortex were required for object localization. Single whiskers were sufficient to perform the task. Mice measured absolute object location with precisions of $<1 \mathrm{~mm}$. Automated whisker tracking from high-speed video revealed both the whisking strategies and the mechanical input to the sensory follicles underlying object localization. This robust head-fixed behavior will facilitate apply- 
Table 1. Mice appearing in this paper

\begin{tabular}{|c|c|c|}
\hline Mouse & Figures including mouse & Pole positions \\
\hline JF3465 & $\begin{array}{l}\text { Figs. } 2 B, 7,12 B, 14 C, D, 15,16 B, 17 B, 18,20 \\
\quad \text { supplemental Fig. } 4^{a}\end{array}$ & Posterior \\
\hline JF3468 & Figs. $2 B, 4 C, 5$ & Posterior \\
\hline JF3470 & Figs. $2 B, 4 C, 5$ & Posterior \\
\hline JF4003 & Fig. $4 \mathrm{C}$ & Posterior \\
\hline JF4004 & $\begin{array}{l}\text { Figs. } 2,4 A, B, 5,6,7,9,12 B, 14 C, D, 15,16 B \\
\qquad 17 B, 18,20 \text {; supplemental Fig. } 4^{a}\end{array}$ & Posterior \\
\hline$J$ J4005 & Fig. $4 \mathrm{C}$ & Posterior \\
\hline JF4793 & $\begin{array}{l}\text { Figs. } 2 B, 3,6 A-C, 7,12 B, 14 C, D, 15,16 B, 17 B \\
18,20 \text {; supplemental Fig. } 4^{a}\end{array}$ & Posterior \\
\hline JF4795 & Fig. 3 & Posterior \\
\hline JF4796 & Figs. $2 B, 3,4 A, 6 A-C$ & Posterior \\
\hline JF4797 & Figs. $2 B, 3,6 C$ & Posterior \\
\hline JF6499 & Figs. $2 B, 3,4 A, 6 D, E$ & Posterior \\
\hline JF6503 & Figs. $2 B, 3,6 D, E$; supplemental Fig. $3^{a}$ & Posterior \\
\hline JF6504 & Figs. $2 B, 3,6 D, E ;$ supplemental Fig. $3^{a}$ & Posterior \\
\hline JF8401 & Supplemental Fig. $2^{a}$ & Anterior \\
\hline JF8402 & Supplemental Fig. $2^{a}$ & Anterior \\
\hline $\mathrm{JF} 8410^{b}$ & $\begin{array}{l}\text { Figs. } 2 B, 11 B, 12 A, 14 A, B, 15,16 A, 17 B, 18, \\
19 E ; \text {; supplemental Fig. } 2^{a}\end{array}$ & Anterior \\
\hline JF8411 & Supplemental Fig. $2^{a}$ & Anterior \\
\hline $\mathrm{JF} 8632^{b}$ & $\begin{array}{l}\text { Figs. } 2 B, 8 B-D, 10,11,12 A, 13,14 A, B, 15,16 A \text {, } \\
\qquad 17 B, 18,19 E ; \text {; supplemental Figs. } 2,5,6^{a}\end{array}$ & Anterior \\
\hline JF9054 ${ }^{b}$ & $\begin{array}{l}\text { Figs. } 2 B, 8 A, 11 B, 12 A, 14 A, B, 15,16 A, 17 B \\
\qquad 18,19 ; \text {; supplemental Fig. } 2^{a}\end{array}$ & Anterior \\
\hline
\end{tabular}

$N=19$ mice. If a mouse appears only in a subset of panels for a figure, those panels are listed.

${ }^{a}$ Available at www.jneurosci.org as supplemental material.

${ }^{b}$ Mouse was used simultaneously for electrophysiology in some behavioral sessions.

ing methods of cellular physiology, including whole-cell recording and two-photon imaging, and provides a foundation for genetic analysis of the underlying neural circuits.

\section{Materials and Methods}

Mice

All mice used in this study were adult (older than postnatal day 60) C57BL/6Crl males (Charles River) (Table 1). Mice were housed singly in cages containing tunnels and bedding material, in a reverse light cycle room that was dark from 9:00 A.M. to 9:00 P.M. All training and behavioral testing occurred during the dark phase. For $\geq 10 \mathrm{~d}$ before training, and on days without behavioral testing, mice were limited to $1 \mathrm{ml} / \mathrm{d}$ water, $\sim 35 \%$ of ad libitum water consumption (Mouse Phenome Database; The Jackson Laboratory; http://www.jax.org/phenome; accession number MPD:92). On days with behavioral sessions, mice generally obtained all water for the day during the session and were allowed to perform until sated. The amount consumed was determined by weighing the mouse before and after the session (including any excrement). The volume consumed fluctuated around $1 \mathrm{ml}$; the precise amount was correlated with how much water the mouse had consumed the previous day. Food was available ad libitum. The weight and health (posture, quality of the fur, and motor activity) of the mice were monitored daily. Mice were occasionally given supplemental water beyond their daily ration of $1 \mathrm{ml}$. These supplements, which were typically $\sim 0.3 \mathrm{ml}$ but ranged up to $1 \mathrm{ml}$, were not generally necessary but were sometimes used in a prophylactic manner to help mice maintain weight. All procedures were in accordance with protocols approved by the Janelia Farm Institutional Animal Care and Use Committee. Three of the 19 mice in this study underwent simultaneous electrophysiological recordings during a subset of behavioral sessions (Table 1); their behavior was indistinguishable as a group from the other mice (data not shown).

\section{Apparatus}

The stimulus object was a $1 / 16$-inch $(\sim 1.59$-mm $)$-diameter pole [stainlesssteel dowel pin; McMaster; part number (P/N) 90145A427] coupled to a linear slider (Schneeberger; P/N NDN 2-50.40), which was driven in the anterior-posterior dimension by a stepper motor with submicrometer resolution (Zaber; P/N NA08B30). This assembly was mounted on a pneumatic linear slider (Festo; SLS-10-30-P-A Mini slide; P/N 170496), connected to a compressed air source, and controlled by a solenoid valve (Festo; CPE 10-M1BH-5L-QS-6; P/N 196883). The pneumatic slider rapidly $(\sim 0.5 \mathrm{~s})$ brought the stimulus into and out of reach of the whiskers under computer control. The pole moved along a trajectory that was at a lateral distance of $9.8 \mathrm{~mm}$ from the midline. The entire apparatus was mounted on a vibration-isolation table (Technical Manufacturing Corporation; P/N 63 533) and enclosed in a custom light isolation box [except for experiments shown in supplemental Fig. 2 (available at www. jneurosci.org as supplemental material), in which case some sessions were conducted in "training rigs" that lacked vibration isolation tables]. The interior of the box was covered with sound isolation foam (McMaster; $\mathrm{P} / \mathrm{N}$ 5692T49). Mice were monitored with an infrared-sensitive video camera (Super Circuits; P/N PC6EX3). Illumination for this camera was either via scattered light from the high-speed video illumination (see below) or with a $940 \mathrm{~nm}$ light-emitting diode (LED) (Roithner Lasertechnik; P/N LED-940-66-60) operated at low currents and without focusing optics.

A custom acrylic "lickport" used to record licks and to deliver water rewards was placed on a manipulator (Newport; P/N MT-XYZ) in front of the mouse, within reach of its tongue. Licks were recorded as interruptions in the light path between an $860 \mathrm{~nm}$ LED (Panasonic; P/N LN77L) and a phototransistor (Fairchild Semiconductor; P/N L14G1). To maximize space around the face for high-speed videography, the phototransistor/LED pair were remote from the lickport but coupled to it using a 1-mm-diameter acrylic fiber optic (Edmund Optics; P/N NT02-544). Water rewards were delivered by gravity into the lickport through a 0.05-inch-diameter steel tube, under solenoid valve control (The Lee Company). To limit the time water remained at the lickport, and to prevent pooling, water was pumped out of the lickport through a 0.0325 inch tube using a peristaltic pump (Markson LabSales).

Puffs of compressed air (typically $10 \mathrm{psi}$ ) for punishment were delivered through a small metal tube $[\sim 2.3 \mathrm{~mm}$ inner diameter (i.d.) $]$ pointed at the face from a distance of several centimeters and were gated by a solenoid valve (Nresearch; P/N HP225T011). This air puff produces a sensation at the back of the human hand approximately like the airstream produced by strong blowing of the breath through a beverage straw at a distance of $\sim 2 \mathrm{~cm}$.

The apparatus was controlled by a software system (Z. Mainen, C. Brody, Cold Spring Harbor Laboratory, Cold Spring Harbor, NY) comprising MATLAB (Mathworks) routines, running on Windows XP, communicating over the ethernet with a real-time control system implemented in C on Linux using the RTLinux (www.rtlinux-gpl.org) or RTAI (www.rtai.org) kernel patches (C. Culianu, Cold Spring Harbor Laboratory, Cold Spring Harbor, NY). The real-time Linux system interfaced with valves and recorded licking responses using a PCI-6025E data acquisition board (National Instruments) and the COMEDI drivers (www. comedi.org). The stepper motor was driven by its controller in response to commands sent through the serial port of the Windows XP computer running MATLAB.

Mice were placed in acrylic (1/1/8 inch i.d.; McMaster; P/N 8486K433) or aluminum (32 mm i.d.) tubes such that their heads extended out the front and they could use their front paws to grip the tube edge (see Fig. $1 \mathrm{~B})$. A surgically implanted head post was immobilized using a custom mount extending either to the front or to the sides of the mice. Mice were thereby head-fixed in a natural crouching position with their whiskers free to move around the space surrounding their heads.

\section{Head post surgery}

For head fixation, a small custom stainless-steel or titanium head post was implanted and mounted in the behavioral apparatus. To implant the head post, mice were anesthetized with isoflurane ( $\sim 2 \%$ by volume in $\mathrm{O}_{2}$; SurgiVet; Smiths Medical). The eyes were covered with a thin layer of petroleum jelly. Bupivacaine $\mathrm{HCl}$ (Hospira; $10 \mu l, 0.5 \%$, s.c.) was applied locally at the incision site. Mice were mounted in a stereotaxic apparatus (Stoelting) and kept on a thermal blanket (Harvard Apparatus) to maintain body temperature. The scalp and periosteum over the dorsal surface 
of the skull were removed. A thin layer of cyanoacrylate adhesive was applied to the skull and covered with dental acrylic (Jet Repair Acrylic; Lang Dental Manufacturing; P/N 1223). Head posts were fixed to the skull using dental acrylic. Buprenorphine $\mathrm{HCl}(0.1 \mathrm{mg} / \mathrm{kg}$, i.p.; Bedford Laboratories) was used for postoperative analgesia. Ketoprofen $(5 \mathrm{mg} / \mathrm{kg}$, s.c.; Fort Dodge Animal Health) was used at the time of surgery and postoperatively to reduce inflammation. Mice were allowed at least $3 \mathrm{~d}$ to recover before water restriction.

\section{Muscimol inactivation}

Muscimol hydrobromide (Sigma-Aldrich; P/N G019) was dissolved in HEPES-buffered artificial CSF (ACSF), pH 7.4, at $5 \mu \mathrm{g} / \mu \mathrm{l}$, aliquoted, and stored at $-20^{\circ} \mathrm{C}$. For injections, mice were lightly anesthetized with isoflurane and kept on a thermal blanket. The eyes were covered with a thin layer of petroleum jelly. Muscimol $(70 \mathrm{nl}$ at $5 \mu \mathrm{g} / \mu \mathrm{l})$ was injected through a hole in the dental acrylic and a small $(<1 \mathrm{~mm})$ craniotomy at a vertical depth of $\sim 300 \mu \mathrm{m}$. The injection system comprised a pulled glass pipette (broken to $\sim 20-30 \mu \mathrm{m}$ i.d.; Drummond Scientific; Wiretrol II Capillary Microdispenser; P/N 5-000-2010) tip-filled with muscimol solution and back-filled with mineral oil. A fitted plunger (manufactured to match the pipette) was inserted into the pipette and advanced to displace the contents using a Narashige hydraulic manipulator (MO-10; Narashige International). The injection pipette was inserted and removed slowly from the left hemisphere of the brain, under control of a Sutter manipulator (MP-285; Sutter Instrument). The injection was performed over the course of $6.3 \pm 2.5$ (mean \pm SD) min. The pipette was left in place for an additional $4.9 \pm 0.9 \mathrm{~min}$ after the injection to prevent backflow. After injection, the craniotomy was covered with bone wax (Surgical Specialties; P/N 901) and a thin layer of dental acrylic. When subsequent injections were made, the dental acrylic and bone wax were removed and the procedure repeated. Stereotactic coordinates were estimated at the time of injections and confirmed post hoc. For barrel cortex injections, coordinates for the three mice were as follows: JF4796, 3.25/ 1.4 (millimeters lateral from midline/millimeters caudal to bregma); JF4793, 2.75/0.5; JF4797, 3.0/1.5; JF4004, 3.4/0.9. Visual cortex injections were as follows: JF4793, 2.0/3.0; JF4004, 2.4/4.1. Experiments began $95 \pm$ $16 \mathrm{~min}$ after the injection. Mice were allowed $77 \pm 16 \mathrm{~min}$ to recover in their cages after waking before beginning the behavioral session. During recovery, mice showed normal locomotor and exploratory whisking activity. Injections of vehicle were identical except that the ACSF lacked muscimol. One of the mice used for muscimol injections (see Fig. 6A-C, square plot symbols) had reduced whisker movements because of an earlier transection of the motor nerve innervating the intrinsic muscles of the right whisker pad; the control condition performance of this mouse did not differ from the others.

\section{Lesions}

Somatosensory cortex lesions were performed on mice already fitted with a head post. Mice were maintained under isoflurane anesthesia on a thermal blanket, as described above. The eyes were covered with a thin layer of petroleum jelly. A craniotomy covering the left barrel cortex was made after removing the overlying dental acrylic. Cortical tissue was aspirated using the tip of a flame-pulled glass Pasteur pipette. Care was taken to avoid aspirating any white matter. The cavity was filled with Gelfoam (Pfizer; P/N 09-0353-01) and then covered with bone wax and finally with dental acrylic. Buprenorphine $\mathrm{HCl}(0.1 \mathrm{mg} / \mathrm{kg}$, i.p.; Bedford Laboratories) was given for postoperative analgesia. Shortly after surgery, all mice exhibited normal locomotor and exploratory whisking activity. Mice were given extra water before and after the surgery $(5-8 \mathrm{ml}$ the day of the surgery, $1-5 \mathrm{ml}$ the day before, 3-5 $\mathrm{ml}$ the day after). Behavioral testing resumed after $4-7 \mathrm{~d}$. In the first mouse tested, three consecutive lesions were made to determine the minimal barrel cortex lesion sufficient to produce a maximal effect on performance. Each surgery was followed by a recovery period and a behavioral session. Only results after the last of these surgeries are shown for this mouse in Figure 6, $D$ and $E$; the three consecutive ablations gave progressive deficits of 85 , 70 , and $47 \%$ correct, versus $92 \%$ correct before surgery. Subsequently, operated mice experienced only a single lesion surgery. After completion of experiments, mice were perfused with PBS followed by $4 \%$ parafor- maldehyde in $0.1 \mathrm{M} \mathrm{PB}, \mathrm{pH}$ 7.4. Coronal sections $(60 \mu \mathrm{m})$ were cut on a vibratome and stained for cytochrome oxidase to confirm the extent of lesions (supplemental Fig. 3, available at www.jneurosci.org as supplemental material).

\section{Whisker trimming}

For most sessions, mice were trimmed to a single row of whiskers or fewer. Whiskers from either row $C$ or row $D$ were spared. Mice used in Figures 4, 7, 9, 12B, 14, $C$ and $D, 16 B$, and 20, and supplemental Figure 4 (available at www.jneurosci.org as supplemental material) were trimmed, where indicated, to row C. Mice used in Figures 8, 10, 11, 12 A, $13,14, A$ and $B, 16 A$, and 19 , and supplemental Figures 5 and 6 (available at www.jneurosci.org as supplemental material) were trimmed to row D. Whiskers were trimmed with scissors under a dissecting microscope, during brief ( $\sim 2 \mathrm{~min}$ ) periods of isoflurane anesthesia. Trimmed whiskers were maintained with periodic cutting at lengths too short to reach the stimulus pole. The whisker trimming depicted in Figure 4 occurred either the afternoon before or the morning of the first posttrim behavioral session. Whiskers were trimmed bilaterally, even though the pole was always presented only to one side of the mouse.

\section{Training and behavioral task}

Training began after head post implantation, recovery, and $\geq 10 \mathrm{~d}$ of water restriction. We did not find it necessary to handle the mice extensively before training. Mice were in most cases briefly anesthetized with isoflurane to facilitate head fixation. We also head-fixed mice without isoflurane and saw no obvious difference in subsequent performance (data not shown). Training began with one to two daily sessions ( $\sim 10-15$ min each) during which the mouse was rewarded for every lick recorded on the lickport (subject to an $\sim 200$ ms minimum interval set by the open time of the reward delivery valve) but were not presented with any stimuli and had no task. By the end of one to two sessions, mice reliably triggered water rewards and consumed water. During the next phase of training, the offset $(D)$ between the go and no-go stimuli was large $(D=8.57 \mathrm{~mm})$ and there was no air puff. At this offset, the no-go stimulus fell slightly anterior to the whisker field when the whiskers were at rest. After mastering this easy version of the task (above $\sim 85 \%$ correct), mice were advanced to the final version, in which the no-go position was moved closer to the go position, $D=4.29 \mathrm{~mm}$ (transition from open to closed symbols in Fig. $3 A$, and from gray to black curves in Fig. $3 B)$. In this configuration, both the go and the no-go stimuli fell within the whisker field. The air puff was introduced after several days of training once mice achieved intermediate performance, that is, once they understood the different stimulus-response requirements but seemed to achieve suboptimal performance because of lack of motivation. Using an air puff from the beginning of training could result in reluctance to make lick responses even on go trials, presumably because the mice did not understand the reason for the punishment (data not shown). An air puff was used with all mice. Mice were trained daily until they achieved a high performance level (see Fig. 3) (>85\% for at least two consecutive sessions). Training was with intact whisker fields. After training, most mice had a subset of whiskers trimmed to near the follicle, as described above.

The sequence for each trial was as follows (see Fig. 1C,D). Trial types (go or no-go) were chosen randomly, subject to the constraint that not more than three consecutive trials of the same type were allowed. Mice did not appear to use information about the limit on trials of the same type [across 12,624 trials in three mice, fraction correct of trials immediately after a run of three, $0.89 \pm 0.007$ (mean \pm SEM); overall fraction correct, $0.90 \pm 0.003]$. The stepper motor positioned the pole for the upcoming trial during the intertrial interval. To minimize possible auditory cues caused by the movement of the pole, the movement sequence comprised a move from the current position to halfway between the go and no-go positions, and finally to the next-trial position. The motor thus always moved an equal distance regardless of whether the upcoming and preceding positions were identical or different. After stimulus positioning, the trial began with the pole descending (time of descent, $\sim 0.5 \mathrm{~s}$ ) into reach of the whiskers. The mouse had until $2 \mathrm{~s}$ from the start of the pole descent to either lick ("go" response) or withhold a lick ("no-go" response). However, licks were only counted as responses if they oc- 
A

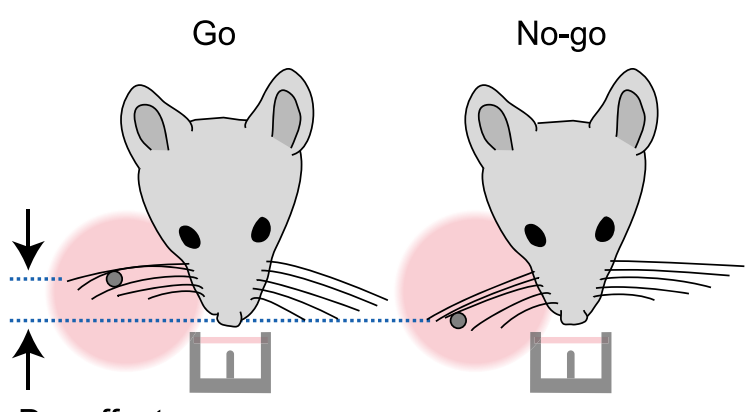

$\mathrm{D}=$ offset

(Anterior - posterior)
B

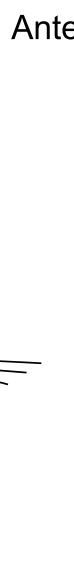

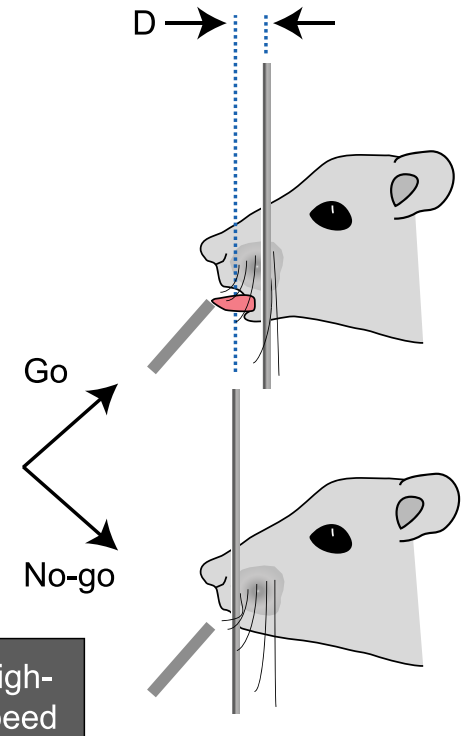

speed

camera

C

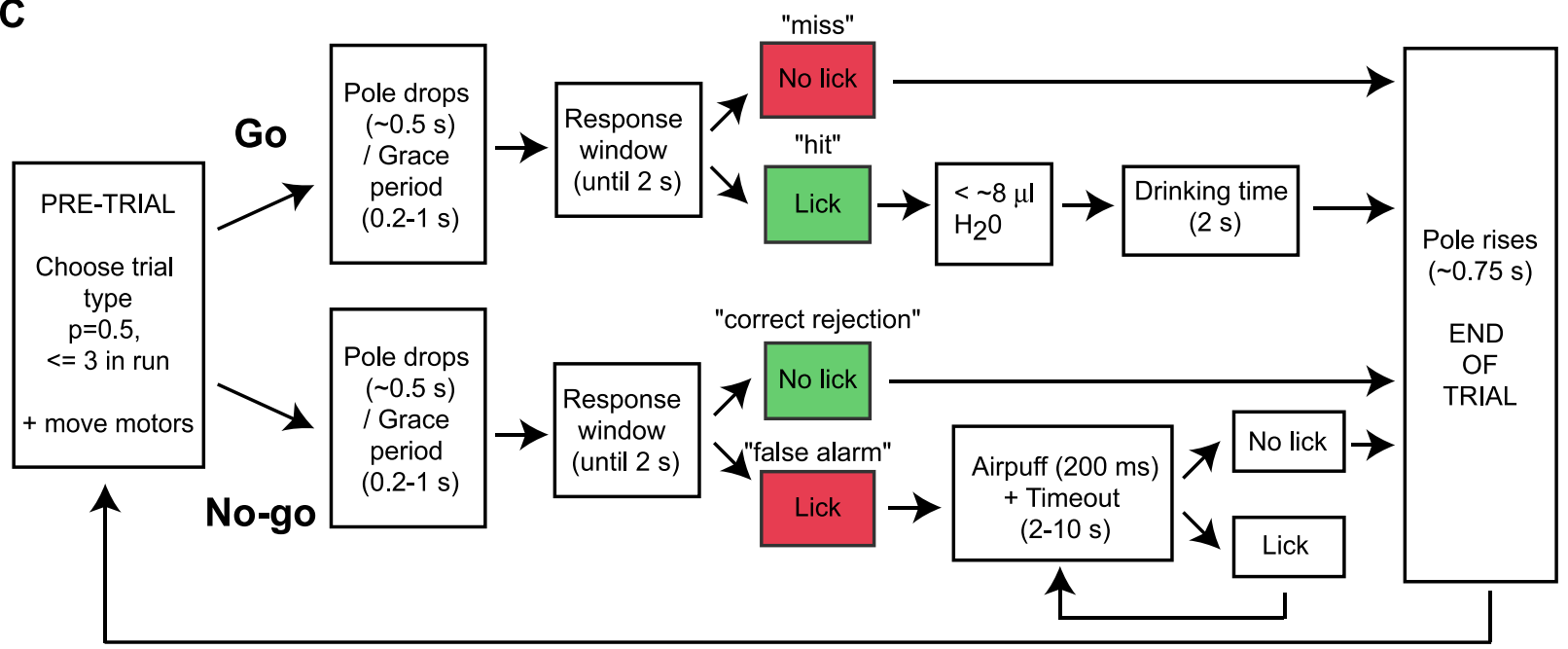

D

Trial N (t=0 s)

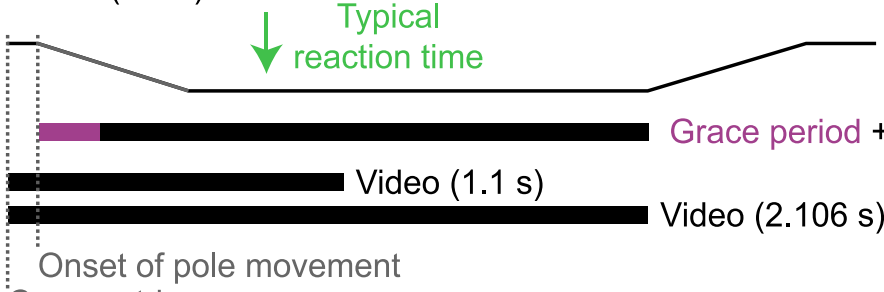

Vertical pole position (Z)<smiles>C=C1CC1</smiles>

Re-position pole (A-P)
Trial $\mathrm{N}+1(\mathrm{t} \approx 6 \mathrm{~s})$

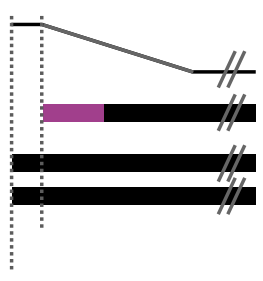

\section{$0.5 \mathrm{~s}$}

Figure 1. Ago/no-go tactile objectlocalization task for head-fixed mice. $A$, Top-view schematic of position of the go (left) and no-go (right) stimuli. A thin pole was presented lateral to the mouse face on one side. The go and no-go positions differed along the anterior-posterior axis. A lickport comprising a water spout for reward delivery and an LED/phototransistor pair for recording lick responses was placed in front of the mouse. The area surrounding the pole and the whiskers was illuminated for high-speed videography with $940 \mathrm{~nm}$ infrared light (shown in pale red). $\boldsymbol{B}$, Side-view schematic showing stimulus geometry. The pole moved in the anterior-posterior axis and also up and down, into and out of reach of the whiskers. Go and no-go trials differed in the anterior-posterior position of the pole. On go trials, mice licked at the lickport; on no-go trials, mice had to withhold licks. The mouse crouched in a natural position inside a tube, with its head in a fixed position in front of the tube (head fixation not shown). The pale red shading indicates high-speed video illumination as in $\boldsymbol{A}$. C, Block diagram of the sequence of events for a single trial (see Materials and Methods). $\boldsymbol{D}$, Schematic timeline of events during a trial. The trial begins with the triggering of either a 1.1 or a 2.106 s high-speed video sequence. Shortly afterward, the pole began its descent into reach of the whiskers. During a short grace period, indicated in purple, any lick responses from the mouse were not used to score the trial (typically, trained mice did not lick during this grace period anyway). The period of time during which the pole is moving into position is indicated by gray shading. 
A

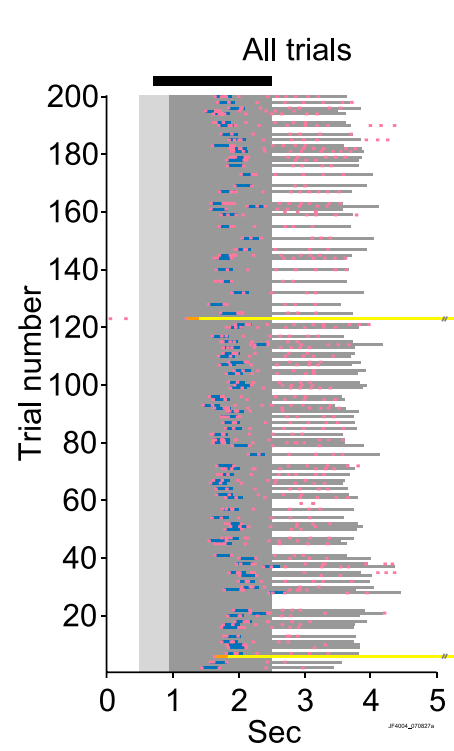

B

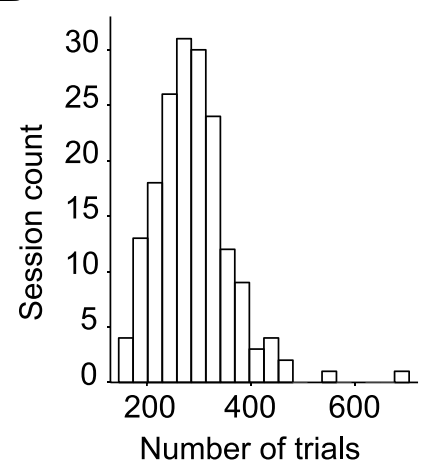

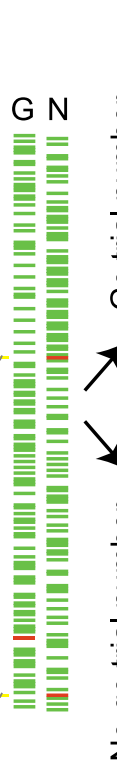

$100_{1}$
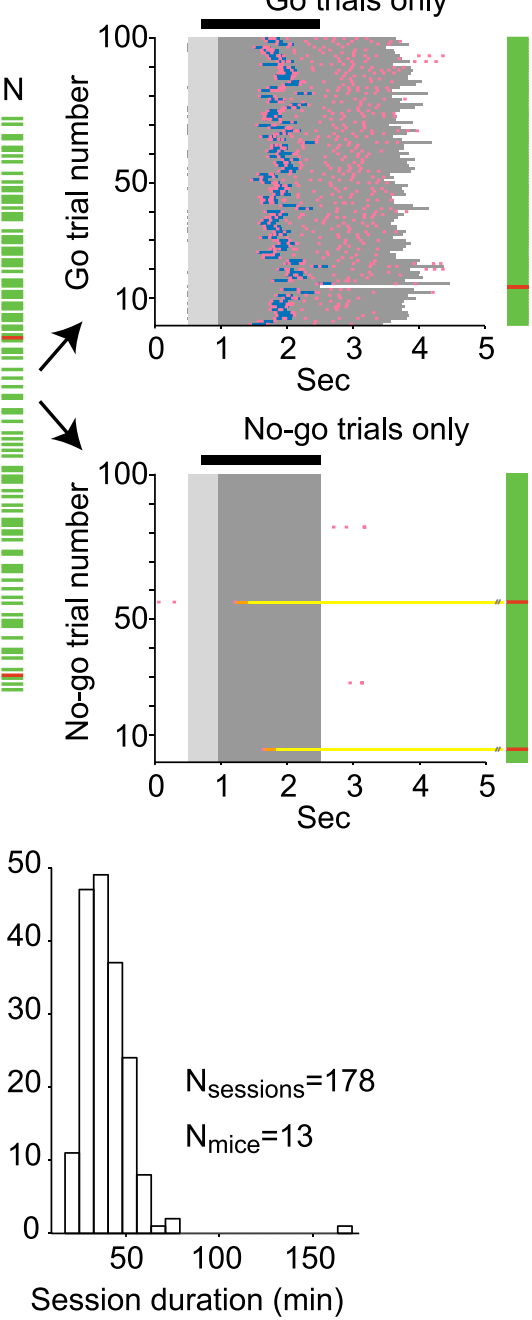

Figure 2. Mice perform at high levels for hundreds of trials. $\boldsymbol{A}$, Raster of events from 200 trials from an example behavioral session. The abscissa shows time from start of trial. The pink tick marks indicate licks (photobeam interruptions). Go and no-go trials are randomly interleaved in the order performed by the mouse (left side) or separated into go and no-go trials (right). The horizontal green and red marks in right columns indicate whether each trial is correct or incorrect, respectively, and on the left raster are separated into two columns corresponding to go (labeled " $\mathrm{G}$ ") and no-go (labeled " $\mathrm{N}$ ") trials. The light gray shading shows approximate travel time of the pole as it descends. The dark gray shading indicates that the pole is fully descended and in reach of the whiskers. The blue bars indicate open times of the reward water valve. The orange bars indicate open time of the air puff valve. The yellow bars show the time-out period and are truncated on the right side for clarity (otherwise extending past $5 \mathrm{~s}$ ). The horizontal black bar at the top of each raster indicates the answer period window (see Materials and Methods). In this session, the mouse performed easy localizations of stimuli separated by $4.29 \mathrm{~mm}$. In simple localizations, trained mice make few licks outside of the appropriate times. $\boldsymbol{B}$, Histograms of number of trials completed per session (left) and session duration (right) for trained mice.

curred in the "answer period," a window that ended at $2 \mathrm{~s}$ and followed a 0.2-1.0 s "grace period" starting at the onset of the pole descent, during which licks had no consequences (see Fig. $1 D$ ). Thus, mice had to either make a lick response within a 1-1.8 s window or withhold licking (as appropriate). The grace period was used primarily as an aid during training. In learning to make a no-go response, some mice would emit a lick immediately on hearing the pole start to move, before it was in reach of the whiskers, but then stop themselves from making any additional licks. If such trials were scored as false alarms, these mice would likely become discouraged. Experienced mice rarely made such premature licks (see Fig. $2 A$ ). The grace period was varied during training to suit the tendencies of individual mice; mice that made more premature licks could be given longer grace periods. Ultimately, all mice had a fixed grace period of either 0.2 or $0.75 \mathrm{~s}$. The reaction time measurements and other data in Figure 20 were all from mice with $0.2 \mathrm{~s}$ grace periods. At this shortest grace period $(0.2 \mathrm{~s})$, mice were able to respond as soon as they could contact the stimulus. After a no-go response, the pole started ascending out of the whisker field exactly $2 \mathrm{~s}$ after starting its descent into the whisker field. Correct no-go responses ("correct rejections") were not rewarded, and incorrect no-go responses ("misses") were not punished. All licks were recorded, but only licks occurring within the answer period were scored as go responses. Correct go responses ("hits") were rewarded with a drop of water $(\sim 8 \mu \mathrm{l})$. However, because the mouse competed for the water with a peristaltic pump, it did not always consume the full $8 \mu \mathrm{l}$. After opening of the water valve, the trial paused for $2 \mathrm{~s}$ to give the mouse time to drink. Incorrect go responses ("false alarms") triggered a $200 \mathrm{~ms}$ air puff and the start of a "time-out" period in which the trial was paused for 2-10 s (typically $5 \mathrm{~s}$; early in training the time-out was occasionally $2 \mathrm{~s}$ ). If the mouse licked during this time-out, it received an identical air puff and the time-out period was restarted. Each trial ended with the pole ascending $(\sim 0.75 \mathrm{~s})$.

\section{Behavioral data analysis}

For measuring learning curves, criterion performance was achieved at the session in which mice performed $>85 \%$ correct with the 4.29 $\mathrm{mm}$ offset, but only if the mouse also performed $>85 \%$ in the next behavioral session. The number of sessions to criterion performance shown in Figure $3 C$ and supplemental Figure $2 B$ (available at www.jneurosci.org as supplemental material) does not include the one to two $(\sim 10-15 \mathrm{~min}$ each $)$ sessions in which the mice learned to lick at the lickport and consume water.

Once a mouse stopped making go responses during a given session, it was considered sated and the experiment was stopped. A number of trials (typically 10) at the end of the session, used to determine that the mouse was sated, were then excluded from analysis. In addition, possibly because of the computer-based trial pacing, we found that it sometimes took mice a few trials before they performed well. We therefore also usually excluded from analysis the first few (1-20) trials. For the learning curves shown in Figure 3 and supplemental Figure 2 (available at www.jneurosci.org as supplemental material), a standard of 20 trials at the beginning and 10 trials at the end were excluded from analysis.

Unless otherwise indicated, performance data in the text are reported as mean $\pm \mathrm{SD}$.

\section{Psychometric curves}

In each session, mice were tested at a single go/no-go position offset (difficulty level) per session. We reasoned that if hard and easy trials were interleaved within a session, the mice might simply wait for blocks of easy trials. By fixing the difficulty for the entire session, performance should be maximized. The order of sessions at different offsets was approximately counterbalanced (supplemental Fig. 4, available at www.jneurosci.org as supplemental material). The minimum intertrial interval depends on the time required to move the motor through the distance separating the go and no-go stimuli. To keep the minimum intertrial interval constant, we programmed the motor to wait until the time required for a $4.29 \mathrm{~mm}$ offset had elapsed in the cases in 
which the actual move time was shorter. Therefore, all trials had the same minimum intertrial interval.

\section{High-speed videography}

High-speed video (500 frames/s; $1 \mathrm{~ms}$ exposure; 8 bit depth) was acquired (AOS X-PRI camera, AOS Imaging Studio software; AOS Technologies) from a bottom view of the whiskers through a Nikon lens (Nikkor AF $50 \mathrm{~mm}$ $\mathrm{f} / 1.8 \mathrm{D} ; \mathrm{P} / \mathrm{N}$ 2137). Illumination for highspeed imaging was from above the whiskers using a $940 \mathrm{~nm}$ LED (Roithner Lasertechnik; P/N LED-940-66-60), delivered through a condenser lens and a ground glass diffuser (Edmund Optics; P/N NT45-655). In some cases, we also illuminated the mouth of the mouse obliquely from below using a second $940 \mathrm{~nm}$ LED with a mounted lens (Roithner Lasertechnik; $18 \mathrm{~mm}$ aspheric glass lens; $f=$ $13.5 \mathrm{~mm}$; P/N LED-Optic-18). Resolution was $\sim 22.68$ pixels $/ \mathrm{mm}$. For the majority of our video sequences, we obtained $1.1 \mathrm{~s}$ movies capturing the period from shortly before the pole began its descent until after the typical reaction time, for 300 consecutive trials from each behavioral session (the number of trials and sequence length being limited by camera memory). In the remaining cases, we obtained longer $2.106 \mathrm{~s}$ movies covering from shortly before the pole began its descent until the end of the answer period window. For these longer videos, we could not obtain consecutive trials because videos were transferred off the camera before triggering the next sequence; instead, a subset of trials was obtained by acquiring as rapidly as possible.

\section{Whisker tracking}

We tracked whiskers to document whisker movements of head-fixed mice while they performed the object localization task with a single row of whiskers. We analyzed the kinematics of whiskers across 3543 movies (most $1.1 \mathrm{~s}$ at 500 fps, 150 pixels by 200 pixels, capturing the whiskers on the stimulus side of the mouse; some movies were $2.106 \mathrm{~s}$ with wider fields of view that captured both the stimulus- and contralateral-side whiskers) using custom software written in $\mathrm{C}$ and Python.

Manual tracking is not feasible for a data set comprising more than a handful of trials. A primary design goal for the algorithms was scaling to large data sets in which comprehensive human validation is not feasible and in which computation time must be considered. For example, video images corresponding to a single behavioral session can amount to more than one million images. In addition, during the object localization task, mice palpated the pole extensively; the whiskers often underwent large changes in curvature $(>0.25 / \mathrm{mm})$, rapid motions (up to $10,000^{\circ} / \mathrm{s}$ ), and "piled up" on the object or crossed one another. Thus, determining shape and position of individual whiskers was more challenging compared with previously treated experimental situations in which animals whisked in air or lightly against surfaces (Knutsen et al., 2005; Voigts et al., 2008). Our method involves several steps: (1) preprocessing, (2) detection of
A
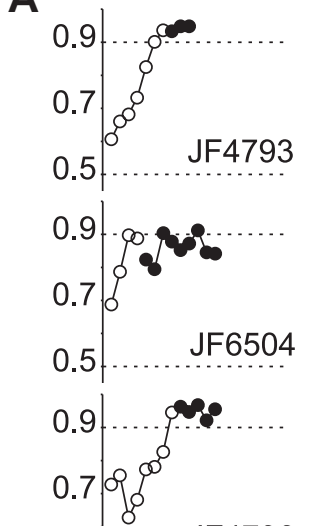

B

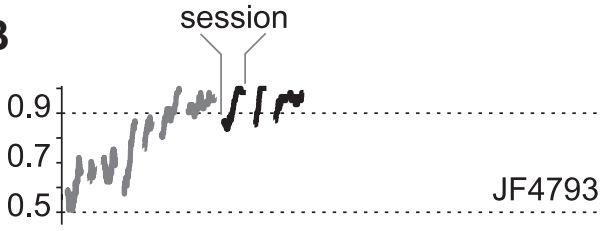

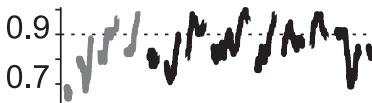

0.5

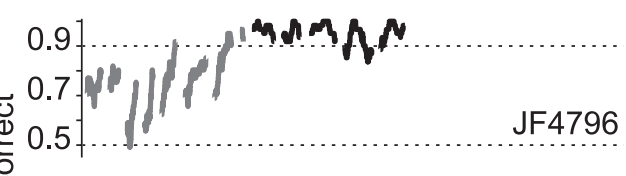

능
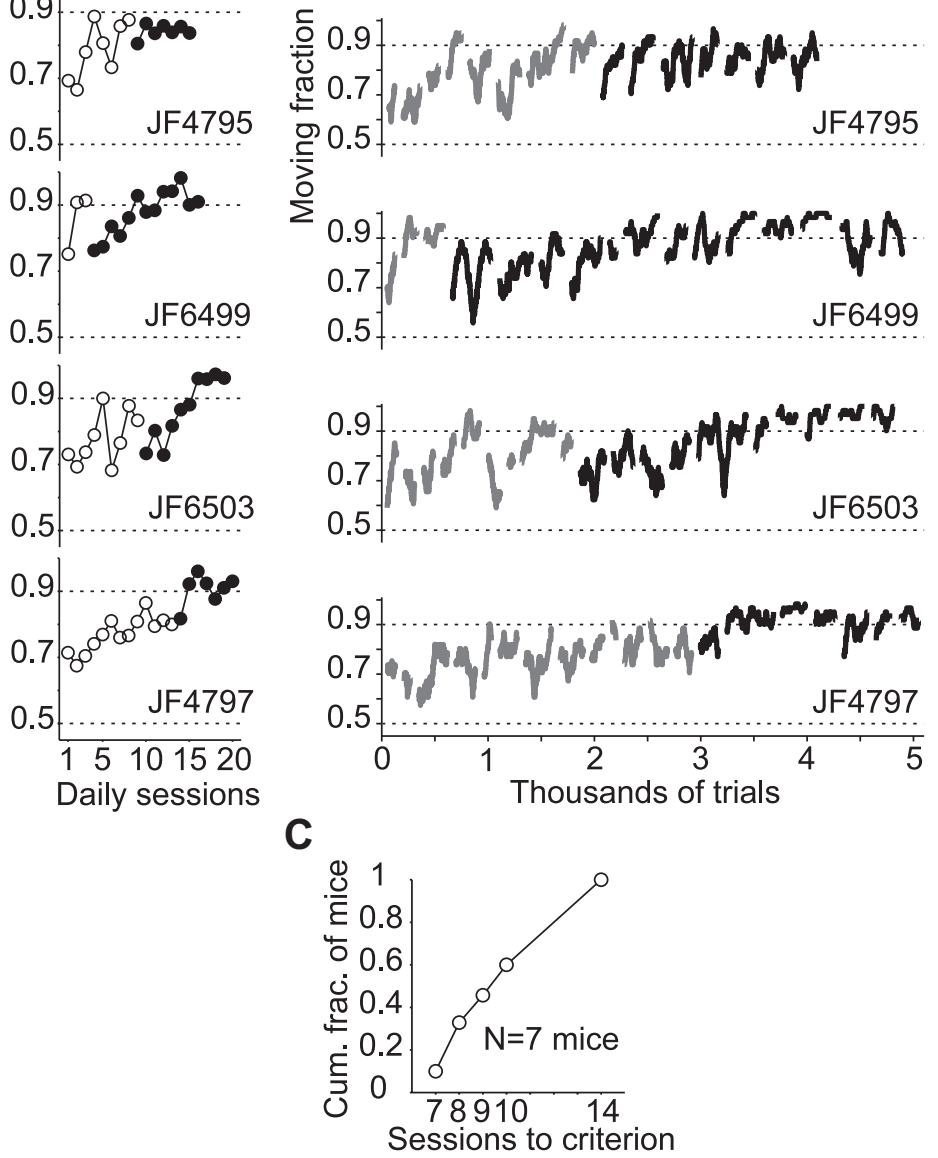

Figure 3. Learning is rapid and occurs both across and within sessions. $A$, Learning curves for a cohort of seven mice. Each data point shows performance averaged over a session. The hollow data points indicate the first stage of training in which go and no-go stimuli were separated by $8.57 \mathrm{~mm}$ (see Materials and Methods). The solid points indicate an easy version of the final task in which stimuli are separated by $4.29 \mathrm{~mm}$. The dashed lines indicate 90 and $50 \%$ correct performance. Before the first data point, mice had one to two sessions ( $\sim 10-15$ min each) of learning to lick at the lickport. $\boldsymbol{B}$, Moving average performance (window of 61 trials) from the same mice and sessions shown in $A$. In several cases, performance increases during the course of an individual session. The gray curves show $8.57 \mathrm{~mm}$ offset sessions and correspond to hollow symbols in $\boldsymbol{A}$. The black curves correspond to the filled symbols in $\boldsymbol{A}$. The dashed lines indicate 90 and 50\% correct performance. Individual sessions correspond to single unbroken curves, separated by small gaps. Gaps reflect the few trials from the beginning of each session that were not analyzed (see Materials and Methods) and 30 trials at the start and end of each session that reflect moving average start-up/ending transients and are not plotted. C, Cumulative histogram of the number of daily localization sessions to an $85 \%$ correct performance criterion across mice. The fastest mouse achieved criterion performance in 7 sessions, and the slowest in 14 sessions.

whiskers, (3) tracing of whiskers, and (4) determination of the identity of traced whiskers.

Preprocessing. For the images collected as part of this study, it was necessary to correct for a systematic multiplicative bias (2-3\%) between 
A

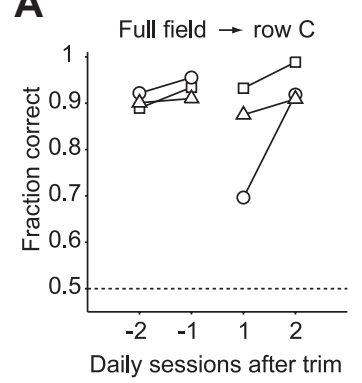

B

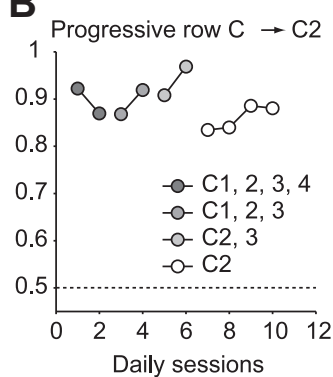

C

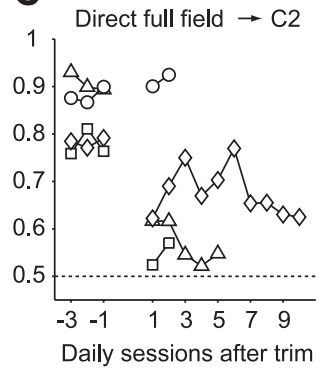

Figure 4. A single whisker is sufficient for object localization. $A$, Performance of mice recovers quickly after trimming from a full whisker field down to row $C$ only. The plot symbols show session-averaged performance for three different mice. Two consecutive sessions before whisker trimming ( $x$-axis ticks at $-2,-1)$, and two consecutive sessions after are shown. By the second session after trimming, performance has recovered to baseline levels. $\boldsymbol{B}$, Performance for one mouse as whiskers are trimmed progressively from row $C$ to $C 2$. Even with a single whisker, the mouse performs at a high level. $C$, Performance of mice after abrupt trimming from a full whisker field to $C 2$. The plot symbols show four different mice for three sessions before trimming ( $x$-axis ticks from -3 to -1 ) and up to 10 sessions after trimming. Mice perform above chance but most ( 3 of 4 ) show a significant decline in performance. Furthermore, three of four mice eventually lost C2. Abrupt trimming from the full whisker field to a single whisker did not therefore result in stable, high performance.

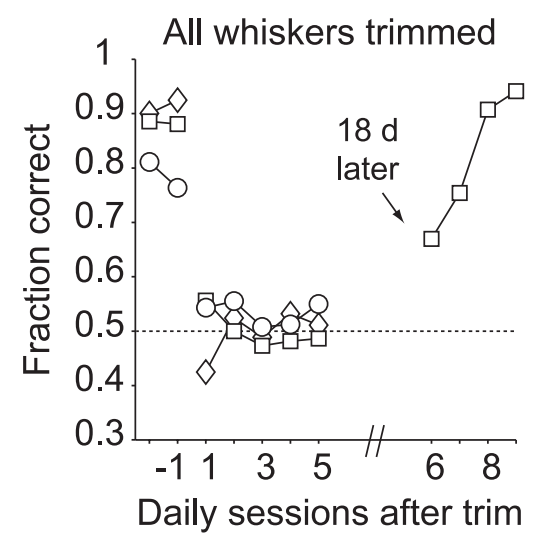

Figure 5. Whiskers are necessary for object localization. After abrupt trimming of all whiskers, performance declines to chance levels. Different plot symbols show three mice for two sessions before $(x$-axis ticks at $-2,-1)$ and five sessions after all whiskers were cut short enough that they did not contact the pole. Chance performance is indicated by the dashed line. Even after five sessions, mice did not use any nonwhisker cues, indicating that our task is whisker dependent. After $18 \mathrm{~d}$ of whisker regrowth, one mouse was tested again; by the third session, performance had reached pretrimming levels.

neighboring pairs of scan lines introduced by the high-speed camera. The ratio of the intensity between a pixel and an above neighboring pixel was computed for each pixel in odd-numbered horizontal lines across a movie. The bias was then measured as the mean of these ratios for pixels within an intensity range chosen to avoid quantization and saturation errors. These were the pixels with intensities above the image mean (150160 typically) and $2 \%$ below saturation (250). Multiplying evenly numbered lines by the measured bias corrected the image, improving downstream analysis.

Detection of whiskers. Initiating sites were found by analyzing the locations of local minima. Along whiskers, local minima lie along a line, whereas elsewhere they do not. In a $5 \times 5$ window about each pixel, local minima were located and the principal direction of their positional variation determined. Computing the fraction of the total variance lying in the principal direction and applying a threshold resulted in 50-100 initiation sites found per whisker, with $10-20$ false positives per image. The threshold was determined empirically by maximizing true positives while minimizing false positives over a representative subset of 10 images. Whiskers were always detected. Each image was analyzed independently to find initiating sites. Alternatively, the initiation site may be chosen manually using a graphical user interface.

Tracing of whiskers. Tracing was started at the initiation sites, removing them as they were traced over. On average, 1.3 traces were initiated per visible whisker. Duplicate traces sometimes occurred and were merged by averaging. Tracing proceeds by estimating the whisker angle locally, and then bidirectionally extending the trace from that point until both ends terminate. The output is a sequence of positions distributed along the center of the whisker.

Tracing is based on a whisker modeled as a rectangular valley in the image, with variable position, width, and angle (supplemental Fig. 1, available at www.jneurosci.org as supplemental material). The center of the whisker is estimated with subpixel precision by finding a position that minimizes the Laplacian of the correlation between the model and the image, or correlating the Laplacian of the model (the line detector) with the image (Torre and Poggio, 1986). Approximating the Laplacian of the model, a line detector was designed as two rectangular, identically oriented, step-edge detectors [ $0.8 \mathrm{~mm}$ long, typically 1 pixel $(40 \mu \mathrm{m})$ wide] positioned parallel to one another and spaced by the detector width (supplemental Fig. $1 A$, available at www.jneurosci.org as supplemental material). The length was chosen to match the expected distance over which highly curved whiskers remained approximately linear. To evaluate the correlation at a given point, it was necessary to compute a pixel representation of the detector by evaluating the area integral of the detector over the square domain of each pixel (supplemental Fig. $1 B$, available at www.jneurosci.org as supplemental material). The value of the correlation at that position is then the dot product between pixels in the image and pixels in the evaluated detector. For efficiency, discrete representations of the detector were pretabulated.

From an initial starting point and angle, traces were extended by making small $(40 \mu \mathrm{m})$ steps in the direction indicated by the angle. At each step, a steepest descent method was used to optimize correlation with the detector as a function of whisker width, angle, and subpixel offset. This was performed over a bounded, discrete parameter space with $2.5^{\circ}$ angular precision and a positioning precision of 0.1 pixel $(4 \mu \mathrm{m})$. Width was estimated to 0.5 pixel $(20 \mu \mathrm{m})$ precision.

Tracing was stopped if correlations dropped below threshold. Additionally, tracing was stopped if one of several criteria indicated the optimization procedure could not be trusted to give an accurate representation of whisker shape beyond that point. This was necessary to handle cases in which whisker segments crossed or were partially occluded by the stimulus. The tests were for large angular change between steps, large left-right asymmetry in the intensity field about the detector, or low mean intensity about the detector. If one of these tests failed, several one pixel steps would be taken along the last trusted direction. If tests and correlations were satisfied at one of these points, normal tracing would resume. Otherwise, the trace was terminated at the last trusted point. Traces shorter than $2 \mathrm{~mm}$ were classified as hairs and rejected.

For steps 1-3 (preprocessing, whisker detection, whisker tracing), images were serially processed at rates in excess of 120 frames/s $\left(3.8 \times 10^{6}\right.$ pixels/s; running on a $2007 \mathrm{Mac}$ Pro 2.1, $3 \mathrm{GHz}$ Quad Core Intel Xeon, 4 GB $667 \mathrm{MHz}$ RAM). Additionally, this software can take full advantage of commonly available multicore PCs to analyze data in parallel processes to achieve near real-time performance.

Determination of the identity of traced whiskers. Although the current implementation of the tracker automatically identifies whiskers (our unpublished data), for this study whisker identity was established using the Python-based user interface and semiautomated specification; every video frame was viewed to confirm proper tracing and to establish whisker identity. 


\section{Whisker data analysis}

After tracking a whisker, the medial axis of that whisker is represented as a sequence of $N$ points, $\left(x_{i}, y_{i}\right)$, providing whisker angle, curvature, and arc length. To overcome discretization noise and compute accurate derivatives, the shape was approximated as a parametric curve, $c(l)=(x(l), y(l))$, where $l \in[0,1]$, and $x(l)$ and $y(l)$ are fifth-degree polynomials. The polynomials were computed by fitting $x_{i}$ and $y_{i}$, respectively, as a function of $l_{i}$, where $l_{1}=0$ and $l_{i}=\sum_{k=2}^{i} \sqrt{\left(x_{k}-x_{k-1}\right)^{2}+\left(y_{k}-y_{k-1}\right)^{2}} / \sum_{k=2}^{N} \sqrt{\left(x_{k}-x_{k-1}\right)^{2}+\left(y_{k}-y_{k-1}\right)^{2}}$. Angle $(\theta$; in degrees), signed curvature ( $\kappa$; in $1 /$ millimeters), and arc length ( $s$; in millimeters) were derived from $c(l)$ at each time point as follows:

$$
\begin{gathered}
\theta(t)=\frac{1}{b-a} \int_{a}^{b} \tan ^{-1}\left(y^{\prime} / x^{\prime}\right) d l, \\
\kappa(t)=\frac{1}{d-c} \int_{c}^{d} \frac{x^{\prime} y^{\prime \prime}-y^{\prime} x^{\prime \prime}}{\left(x^{\prime 2}+y^{\prime 2}\right)^{3 / 2}} d l, \\
s(l)=\int_{0}^{l} \sqrt{x^{\prime 2}+y^{\prime 2}} d l,
\end{gathered}
$$

where the intervals $[a, b]$ and $[c, d]$ denote regions of interest over which to average, and $x^{\prime}, y^{\prime}$ denote derivatives with respect to $l$. Note that these intervals are defined in terms of $s(l)$ (see Fig. $8 C, D$ ).

$\theta$ gives the azimuthal angle computed with respect to the midline. Protraction corresponds to increasing angle values. $\theta=0$ is perpendicular to the midline of the mouse. Whisker angle time series $[\theta(t)]$ were smoothed with a $10 \mathrm{~ms}$ moving average, except for those used to compute velocities (see Figs. 14, 15, 20 B) in which unsmoothed time series were median filtered (10 ms window) before calculating velocity.

Whisker follicle position coordinates (see Fig. 19) were estimated by linearly extrapolating past the end of the tracked whisker. The distance to extrapolate was chosen such that the end point was slightly past the fur-lined edge of the bottom view of the face. Follicle position along the path of whisker pad translation $\left(F_{0}\right)$ (see Fig. 19C,D) was measured as the arc length distance along a curve fitted to the set of all follicle coordinates. For the data in Figure 19E, only follicle movement along the anteriorposterior axis was estimated; occlusion of the whisker pad by the lickport prevented estimating medial-lateral movement. For display (see Fig. $19 C, D)$, follicle position was smoothed with a $10 \mathrm{~ms}$ moving average.

Presence or absence of whisker-pole contact (see Fig. 12) was determined by manual inspection of raw video.

\section{Results}

\section{Head-fixed mice perform absolute localization judgments}

We trained head-fixed mice in a go/no-go vibrissa-based object localization task. Mice reported the presence of a vertical pole within a target position (the "go stimulus") or in a distracter position (the "no-go stimulus") by either licking (go response) or withholding licking (no-go response) (Fig. 1A,B; supplemental Movies 1-3, available at www.jneurosci.org as supplemental material). In each trial, the pole was presented at a single location (of two possible locations for that session), and the mouse had to make a decision based on its memory of the positions. Our task therefore probes memory-guided object localization in the laboratory reference frame.

Trials began with the pole above and out of reach of the whiskers. Under computer control, the pole descended rapidly $(\sim 0.5 \mathrm{~s})$ into reach of the whiskers, whereupon the mouse used its whiskers to determine the pole location and choose the appropriate response. Mice had until $2 \mathrm{~s}$ from the onset of pole descent to make a
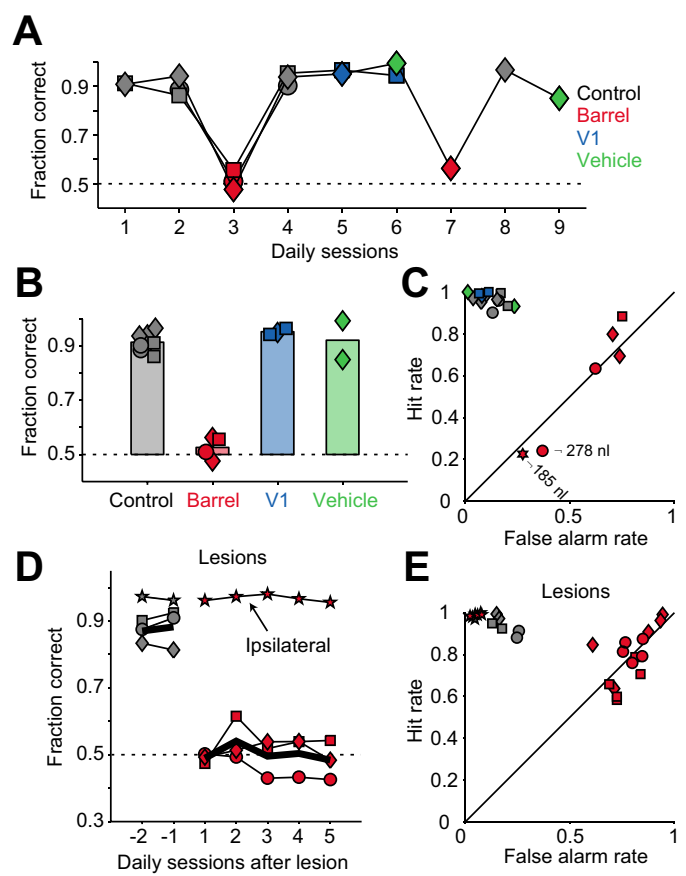

Figure 6. Contralateral somatosensory cortex is necessary for object localization. $\boldsymbol{A}$, Time series showing performance across several consecutive daily sessions in which muscimol was injected or control experiments were performed. The plot symbol shapes indicate three different mice. The gray fill color indicates control sessions with no injections. The red fill indicates muscimol injections into barrel cortex $(70 \mathrm{nl} ; 5 \mu \mathrm{g} / \mu \mathrm{l})$. The blue fill indicates injections of muscimol (70 nl; $5 \mu \mathrm{g} / \mu \mathrm{l}$ ) into primary visual cortex (V1). The green fill indicates injections of saline vehicle (with no muscimol) into barrel cortex. After one to two sessions of control experiments, muscimol was injected into barrel cortex (see Materials and Methods) and performance decreased to chance levels. The following day, performance recovered to baseline levels. Control injections of either saline vehicle into barrel cortex or muscimol into primary visual cortex produced no change in performance. $\boldsymbol{B}$, Bar graph showing the same data as in $\boldsymbol{A}$, but collapsed across time. The plot symbol shapes again indicate different mice. $\boldsymbol{C}$, Hit rate plotted against false alarm rate shows that performance decrements after muscimol injection into barrel cortex result from both an increase in false alarm rate and a decrease in hit rate. The diagonal indicates chance performance. Two additional plot symbols marked "185 $\mathrm{nl}$ " and " $278 \mathrm{nl}$ " indicate that larger volumes of $5 \mu \mathrm{g} / \mu \mathrm{l}$ muscimol depress overall lick rate; these data are not included in $\boldsymbol{A}$ and $\boldsymbol{B}$. The plot symbol shapes and fill colors are as in $\boldsymbol{A}$ and $\boldsymbol{B}$. D, Performance drops to chance levels in mice after aspiration lesions to somatosensory cortex contralateral to the pole stimulus. The plot symbols show different mice. The plot shows two sessions before lesioning ( $x$-axis ticks at $-2,-1$; gray fill color) and five sessions after lesioning (red fill), during which performance does not recover. In one mouse (indicated by the star plot symbols and the arrow), the lesion was made on the stimulus (ipsilateral) side and produced no deficit. The thick black lines indicate average performance across mice with contralateral cortex lesions. $\boldsymbol{E}$, Plotting hit rate against false alarm rate for the data in $\boldsymbol{D}$ shows that performance deficits were attributable to changes in both hit rate and false alarm rate, but mainly to the latter. The plot symbol shapes and fill colors are as in $\boldsymbol{D}$.

response (Fig. 1C,D) (for details, see Materials and Methods). The period before and including the typical response time was captured on high-speed video (Fig. $1 D$ ). Because there were two types of trial (go and no-go) and two possible responses (lick and no-lick), four trial outcomes were possible (Fig. 1C). On "hit" trials, mice made a correct go (i.e., lick) response to a go-position stimulus and were rewarded with a water reward. On "miss" trials, mice failed to make a lick response within the response window. Miss trials were neither punished nor rewarded. On "correct rejection" trials, mice withheld licking in response to a no-go stimulus. Correct rejections were not rewarded. Finally, on "false alarm" trials, mice incorrectly licked on a no-go trial. False alarms were punished with an air puff and a brief time-out. In general, trained mice performed at very high levels (typically 
A Angular diff. (deg)
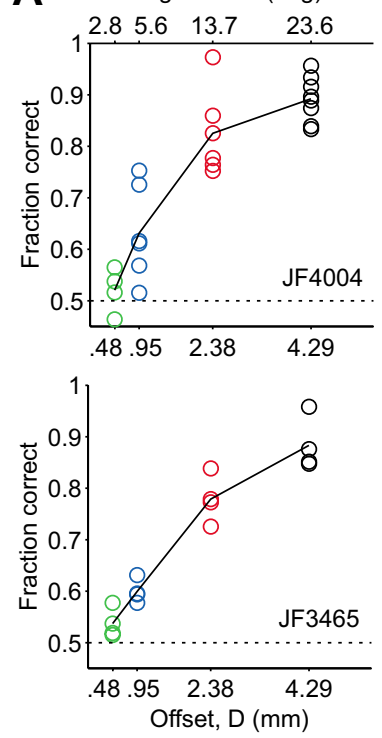

Angular diff. (deg)
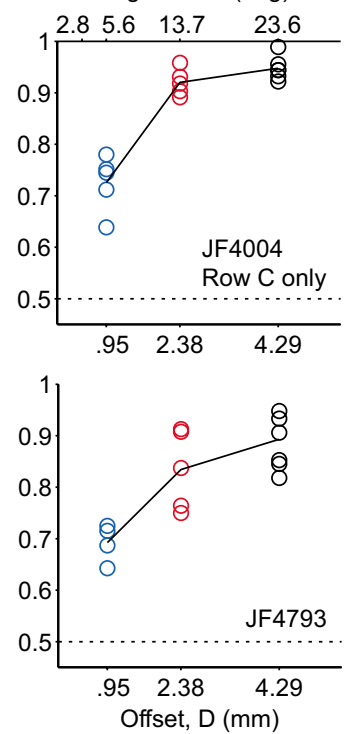

B
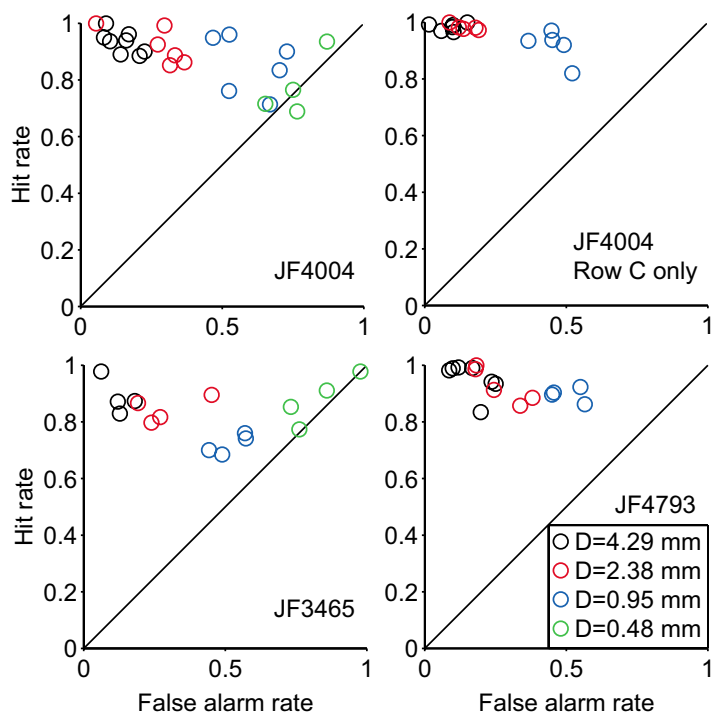

Figure 7. Mice make absolute (memory-guided) azimuthal localizations to better than $6^{\circ}$. $\boldsymbol{A}$, Psychometric curves for three mice relating offset between go and no-go stimulus positions to performance. For one mouse (JF4004), curves were taken separately with all whiskers and with row ( whiskers only. The pole stimulus was moved along the anterior-posterior axis. Approximate azimuthal angular differences corresponding to each offset are shown on the top abscissa. Each data point shows the average performance over a session. Curves connect the means for each offset. The colors indicate different offsets and are the same as in $\boldsymbol{B}$. All mice are above chance at the $0.95 \mathrm{~mm}\left(5.6^{\circ}\right)$ offset. One mouse (JF3465) performs above chance level at the $0.48 \mathrm{~mm}\left(2.8^{\circ}\right)$ offset, although this is marginally significant $(p=0.0625)$. $\boldsymbol{B}$, Plots of hit rate against false alarm rate show that decreases in performance at smaller offsets were attributable both to a decrease in hit rate and an increase in false alarm rate, but mainly to the latter. The diagonal indicates chance performance.

$\sim 90 \%$ correct or better) (Fig. 2 A). Mice made few false alarms and misses when performing easy localizations (Fig. 2A), although false alarms were relatively more common (data not shown). Mice routinely performed hundreds of trials per session, and sessions typically lasted $\sim 40 \mathrm{~min}$ (Fig. $2 \mathrm{~B}$ ).

\section{Learning is rapid and occurs both across and within sessions} Mice learned the task rapidly (Fig. 3). In one cohort of seven mice that were each trained in daily sessions on the same apparatus, the first mouse reached an $85 \%$ correct criterion after $7 \mathrm{~d}$ of training, and the last mouse achieved this criterion within $14 \mathrm{~d}$ (Fig. 3C). A separate cohort of six mice was trained under less ideal circumstances in which mice were moved among training rigs (with slight differences in the position of stimuli); in addition, a 1 month gap without training was interjected. These mice achieved criterion performance within 11-18 d (supplemental Fig. 2, available at www.jneurosci.org as supplemental material). Performance improved within single sessions (Fig. $3 B$ ), as well as across sessions (Fig. $3 A, B$ ).

\section{A single whisker is sufficient for object localization}

To identify whiskers unambiguously using high-speed videography (see below), as well as to limit the number of parallel channels of information the mouse can use to solve the task, it is desirable to have mice perform the task with a subset of whiskers. We therefore tested mice on an easy localization task (size of the offset $D=4.29 \mathrm{~mm}$ ) before and after various patterns of whisker trimming (Figs. 4, 5).

Trimming whiskers from a full whisker field down to row $\mathrm{C}$ caused only a minor reduction in performance (Fig. 4A) (92 \pm $2 \%$ correct for two sessions each before trimming; $94 \pm 4 \%$ correct for second session after trim; $N=3$ mice). In one mouse, we further trimmed progressively down to a single whisker. This mouse maintained a high performance level even for a single whisker (Fig. $4 B$ ). In four mice, trimming from a full whisker field directly to whisker C2 was less successful (Fig. 4C). In three of four mice, performance decreased significantly after trimming ( $82 \pm 6 \%$ over 9 sessions total before trimming vs $63 \pm 7 \%$ correct over 17 sessions total after trimming), whereas performance remained high for the fourth mouse $(88 \pm 1 \%$ over three sessions before trimming vs $91 \%$ correct over two sessions after trimming). However, mice tended to lose the remaining whisker, perhaps through excessive grooming (reflected in the data in Fig. $4 C$; two mice only have two data points after trimming). Therefore, head-fixed mice can use a single whisker for object localization, indicating that the brain combines information about position of the whiskers and contact between whisker and object to compute object distance (Knutsen et al., 2006; Mehta et al., 2007; Diamond et al., 2008).

\section{Whiskers are necessary for object localization}

To exclude the possibility that cues other than vibrissa-based somatosensation contribute to object localization, we trimmed all whiskers to lengths that were too short to reach the pole. Before trimming, these mice performed at a high level ( $86 \pm 6 \%$ correct; $N=3$ mice; $n=6$ sessions total) (Fig. 5 ). After trimming, performance dropped to chance levels ( $51 \pm 3 \%$ correct; $N=3$ mice; $n=15$ sessions). Even after five sessions of training, the mice were unable to use the remaining cues to solve the task (Fig. 5 ). One mouse was retested after $18 \mathrm{~d}$ of whisker growth, which was sufficient for the whiskers to reach the pole. This mouse immediately performed above chance and quickly resumed pretrimming levels of performance (Fig. 5). Thus, mice solve the object localization task with their whiskers.

\section{Somatosensory cortex is required for object localization}

We tested whether our object localization task depends on the barrel cortex. In three mice, we reversibly inactivated barrel cortex using injections of the $\mathrm{GABA}_{\mathrm{A}}$ receptor agonist muscimol (Fig. 6). Performance in control sessions before muscimol injec- 
A

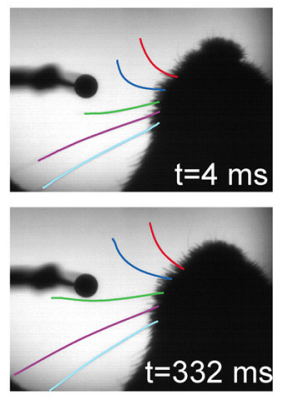

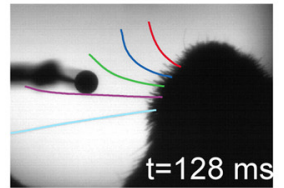

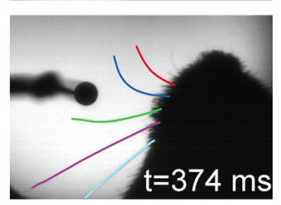

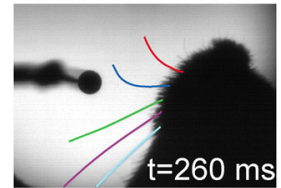

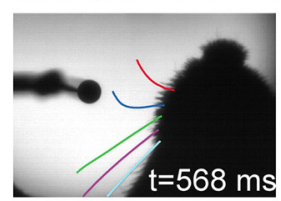

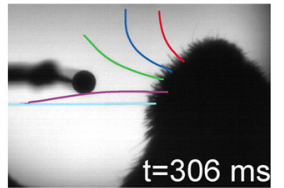

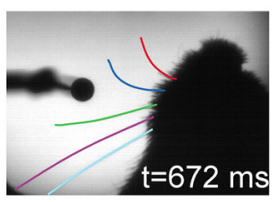

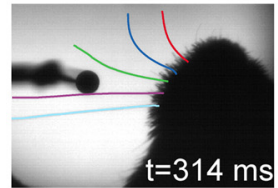

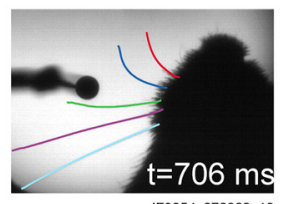

B
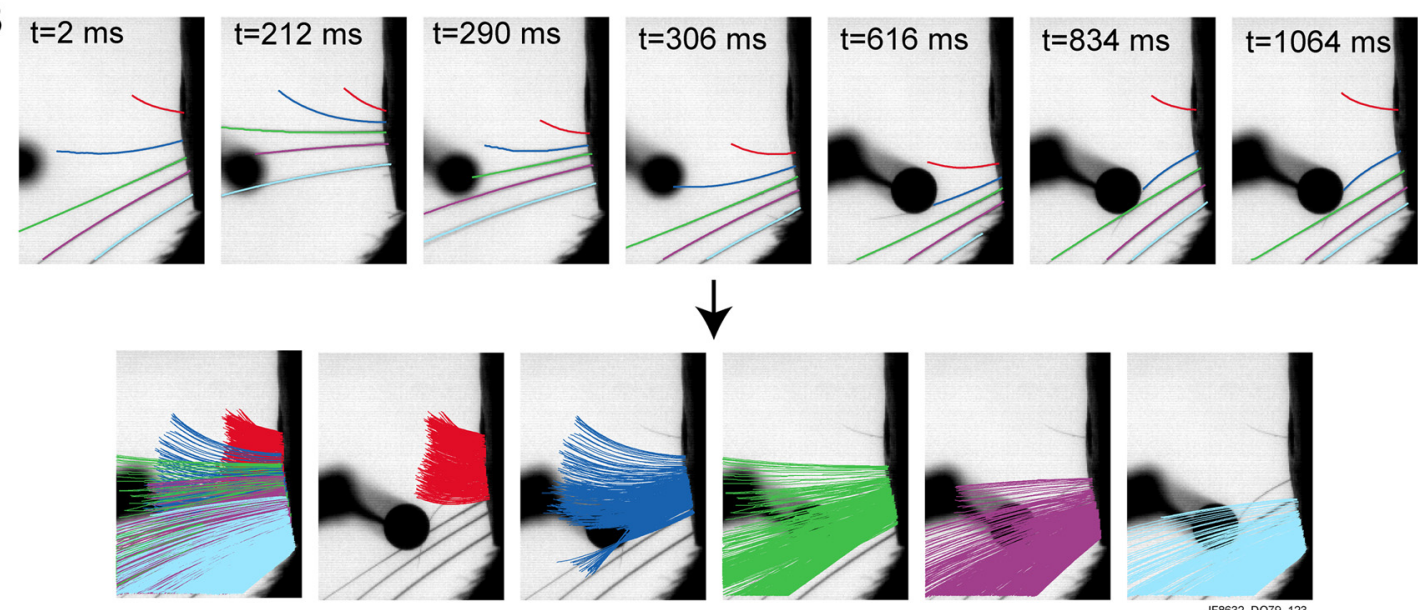

C

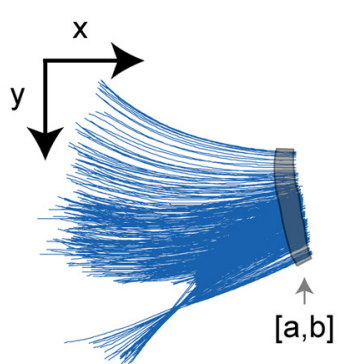

For each frame, average $\theta$, к over an arc length region of interest:
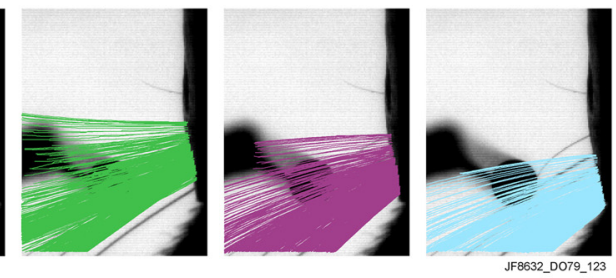

D $\theta(t)=\frac{1}{b-a} \int_{a}^{b} \tan ^{-1}\left(y^{\prime} / x^{\prime}\right) d l$
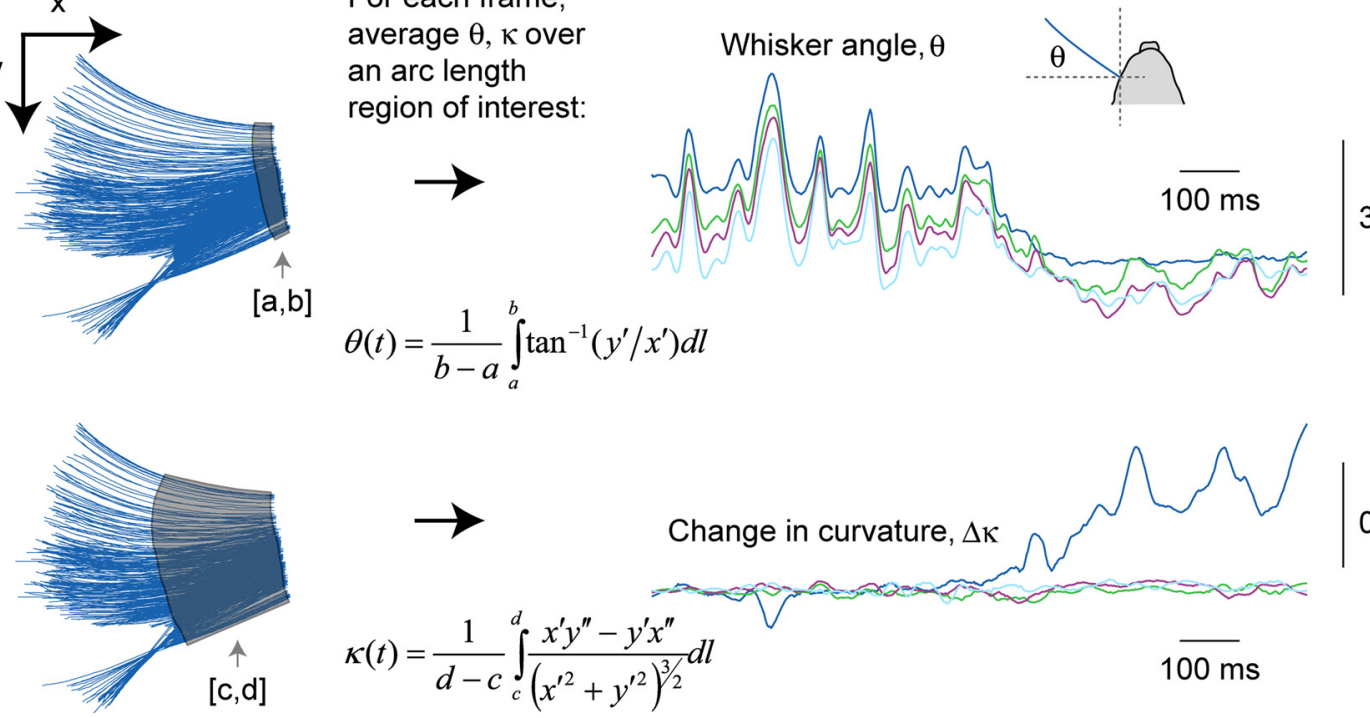

$0.2 \mathrm{~mm}^{-1}$

Figure 8. Extracting azimuthal angle and curvature from high-speed video of whiskers. A, Tracking whiskers in head-fixed mice. Images show a bottom view of the pole stimulus and the mouse. The mouse has a single row of whiskers that were tracked with custom software (see Materials and Methods). The lickport has been moved away and the field-of-view expanded to show the geometry of the pole, mouse head, and whiskers. $\boldsymbol{B}$, Video showing the lickport in place and zoomed to the field-of-view used for the majority of videos (different mouse and session from $\boldsymbol{A}$ ). Single video frames (top row) show the pole descending, as well as changes in the position of the whiskers and curvature change in one whisker (blue) that contacts the pole. The bottom row depicts projections through all video frames. Whiskers are plotted together (left image) or individually (right five images), superimposed on an arbitrary frame from the video. C, Azimuthal angle $(\theta)$ was computed over a small arc length region of interest near the whisker base for each frame (see Materials and Methods). Choice of the region of interest is illustrated schematically for a single whisker (D4; blue) at left. Angle for several whiskers is shown as a function of time for a single trial at right. $D$, Signed curvature $(\kappa)$ was computed over an arc length region of interest for each frame for a given whisker (see Materials and Methods). The region of interest for measuring curvature was longer than that used for measuring angle. Choice of region of interest is illustrated at left. At right, change in curvature $(\Delta \kappa)$ as a function of time is shown for several whiskers. $\Delta \kappa$ was computed as curvature minus the mean curvature in the first $100 \mathrm{~ms}$ of the trial. Each $\Delta \kappa$ trace has been smoothed with a $50 \mathrm{~ms}$ second-order Savitsky-Golay filter. Data in $\boldsymbol{B}-\boldsymbol{D}$ are all from the same trial.

tions was high $(90 \pm 3 \%$ correct; $N=3$ mice; $n=5$ sessions total $)$ (Fig. $6 \mathrm{~A}-\mathrm{C}$ ). After injection of $70 \mathrm{nl}$ of $5 \mu \mathrm{g} / \mu \mathrm{l}$ muscimol into barrel cortex, contralateral to the pole stimulus, performance dropped to chance levels ( $53 \pm 4 \%$ correct; $N=3$ mice; $n=4$ sessions) (Fig. 6A-C). On days after muscimol injections, performance had fully recovered ( $94 \pm 2 \%$ correct; $N=3$ mice; $n=4$ sessions). Injections of saline vehicle into the barrel cortex (92\% correct; $N=1$ mouse; $n=2$ sessions) or muscimol into the 

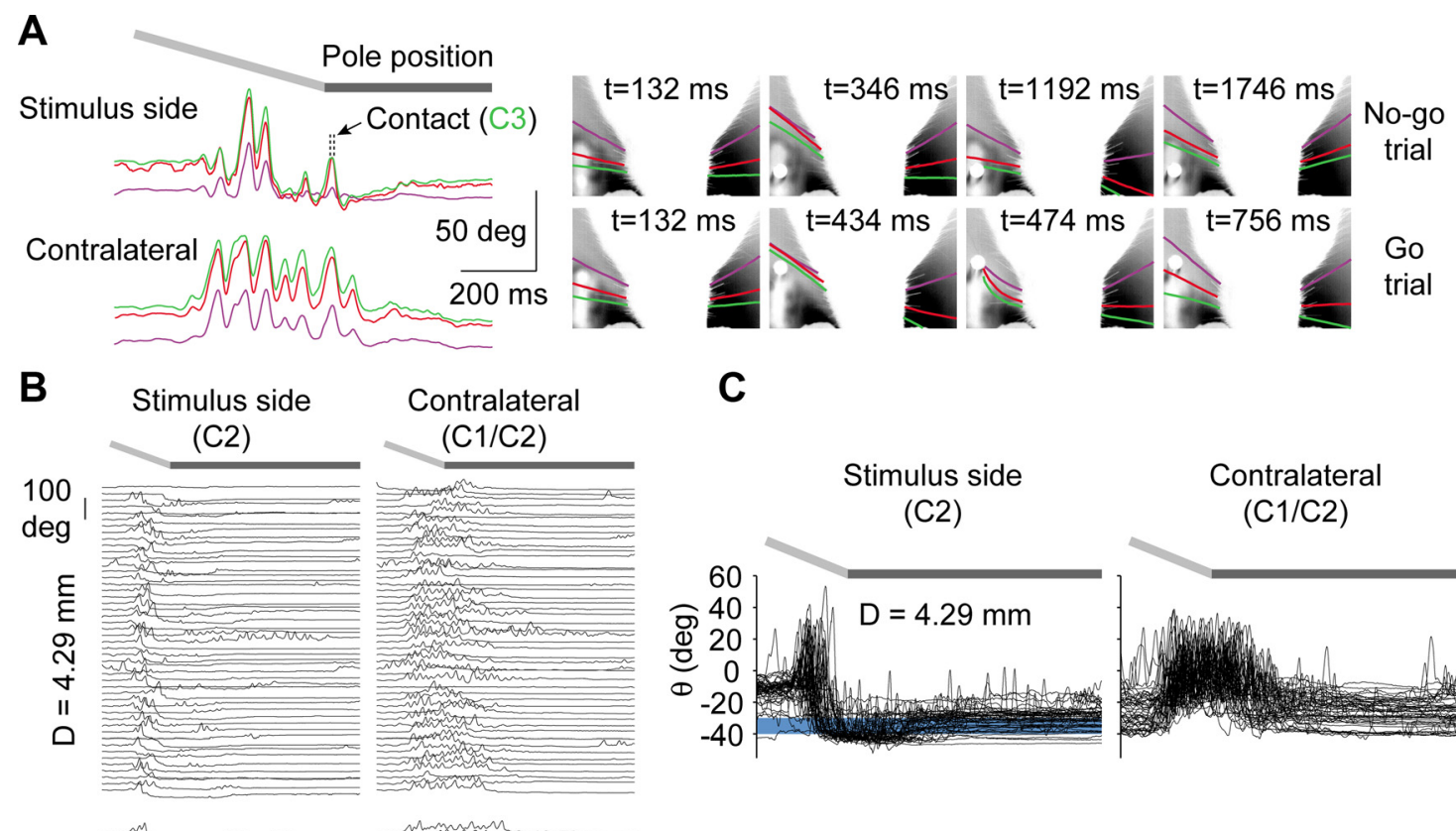

\section{C}
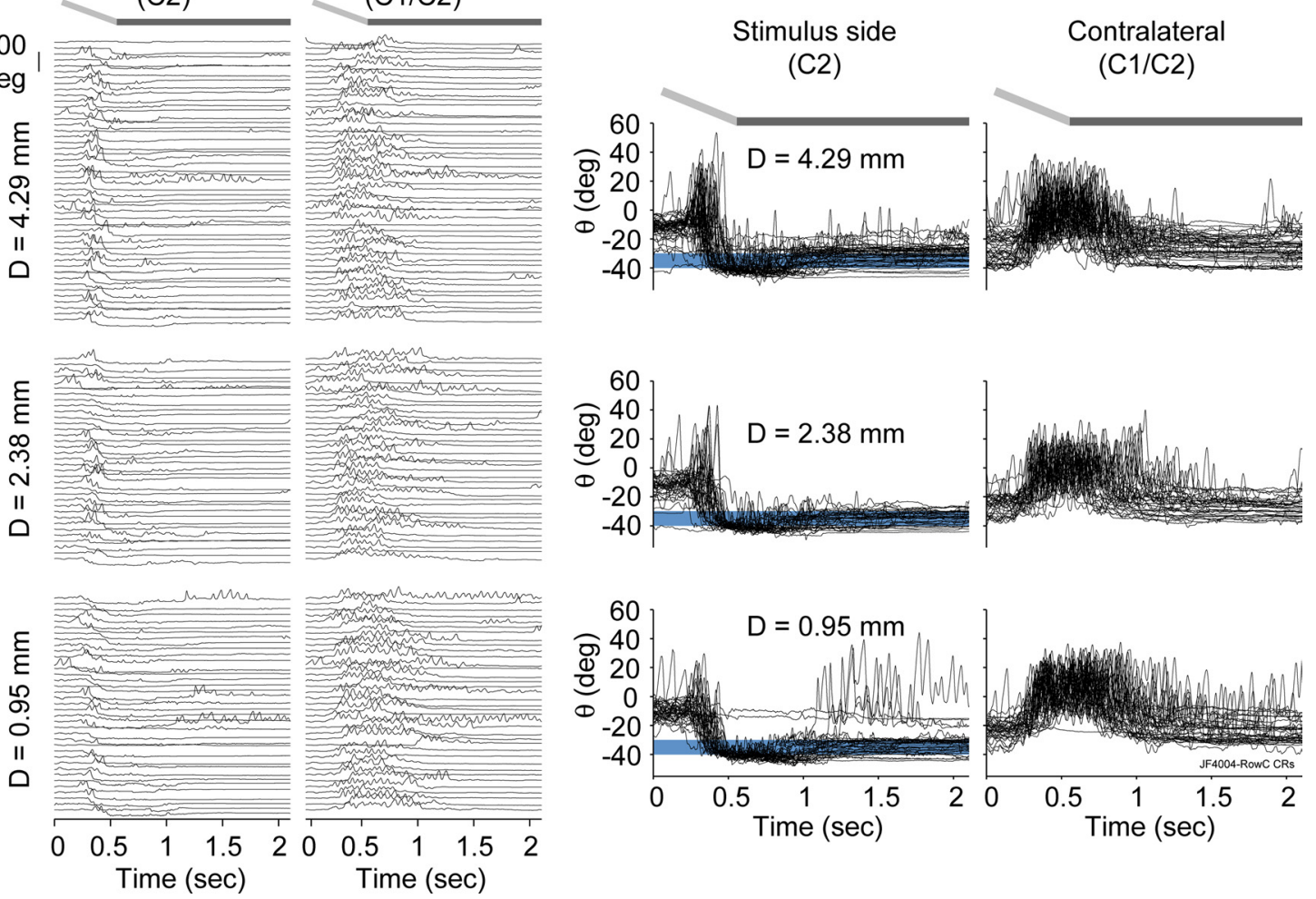

Figure 9. Whisking can be highly asymmetric and is directed to the region of the rewarded ( $g 0$ ) stimulus. $A$, Example whisker angle traces for three tracked whiskers from one trial on the stimulus side of the mouse (left, top traces) and on the contralateral side (left, bottom traces). The light gray slanted bar at top indicates approximate travel time of the pole on its descent. The dark gray horizontal bar indicates that the pole is in its bottom position, within reach of the whiskers. A moment of contact between whisker $\mathrm{C} 3$ and the pole is indicated by the arrow (top traces). Whisking is highly asymmetric between the stimulus and contralateral sides. Movie frames at right highlight asymmetric search strategy of the mouse on a no-go trial (right, top row of frames; same trial shown in traces at left) and a go trial (right, bottom row of frames). In both the no-go and the go trials, the mouse retracts its whiskers on one side to search the position where the rewarded (go) stimulus occurs, whereas the contralateral whiskers make unrelated protractions and retractions. $\boldsymbol{B}$, Rasters of whisker angle traces for the stimulus side (left column) and contralateral side (right column) whiskers for one mouse across many no-go trials and three levels of difficulty (go/no-go position offsets of $D=4.29,2.38$, and 0.95 mm, separated by vertical gaps). Horizontally aligned pairs of traces in the stimulus and contralateral rasters correspond to the same trial. Trials were acquired across several behavioral sessions. The order of trials in the raster was randomized within the three difficulty groups. For the stimulus side, all traces show the position of whisker $\mathrm{C} 2$. In some trials on the contralateral side, whisker $\mathrm{C}$ was traced instead of $\mathrm{C} 2$ because $\mathrm{C} 2$ protracted far enough that it left the field of view. Slanted and horizontal gray bars at top indicate pole travel time and position, as described for $\boldsymbol{A}$. Whisking on the stimulus and contralateral sides is highly asymmetric, with more cycles of protraction and retraction on the contralateral side. C, Data from $\boldsymbol{B}$ at higher zoom, with traces from different trials plotted on top of each other to reveal whisking strategy. On the stimulus side, after a brief protraction the whiskers retract and selectively explore the region of the go stimulus (whose approximate angular position is indicated by the blue bar underneath the whisker traces), even though all traces are from no-go trials. On the contralateral side, whiskers protract and retract for several cycles around a slightly protracted set point. The slanted and horizontal gray bars at top indicate pole travel time and position, as described for $A$. No-go position stimuli (data not shown) are located $\sim 23.6,13.7$, and $5.6^{\circ}$ more protracted (positive) than the indicated go-position stimuli for the $D=4.29,2.38$, and $0.95 \mathrm{~mm}$ offsets, respectively. Data in $\boldsymbol{A}$-C are from a single mouse (JF4004) trimmed to a single row (C) of whiskers. Traces from this mouse were the most consistently asymmetric of the three mice in which we tracked whiskers on both sides of the head. The other two mice, although less stereotyped than the mouse shown here, also showed this same basic asymmetric pattern (for average whisker position traces for two additional mice, see Fig. 200).

primary visual cortex (approximately an equal distance from somatosensory thalamus as the barrel cortex injections; $95 \pm 1 \%$ correct; $N=2$ mice; $n=3$ sessions) did not compromise performance (Fig. $6 A-C$ ).

In four mice, we performed barrel cortex lesions (see Materials and Methods) (supplemental Fig. 3, available at www. jneurosci.org as supplemental material). Three mice received a lesion contralateral to the stimulus, which reduced performance to near chance levels $(88 \pm 4 \%$ correct for two sessions each before lesion vs $50 \pm 5 \%$ correct over five sessions each after lesion; $N=3$ mice) (Fig. $6 D, E$ ), even after five sessions after the lesion. One mouse received an ipsilateral lesion, which did not compromise performance ( $97 \%$ correct for two sessions before vs $97 \pm 1 \%$ correct for five sessions after lesion) (Fig. $6 D, E$ ). Thus, contralateral but not stimulus-side somatosensory cortex is required for object localization. 


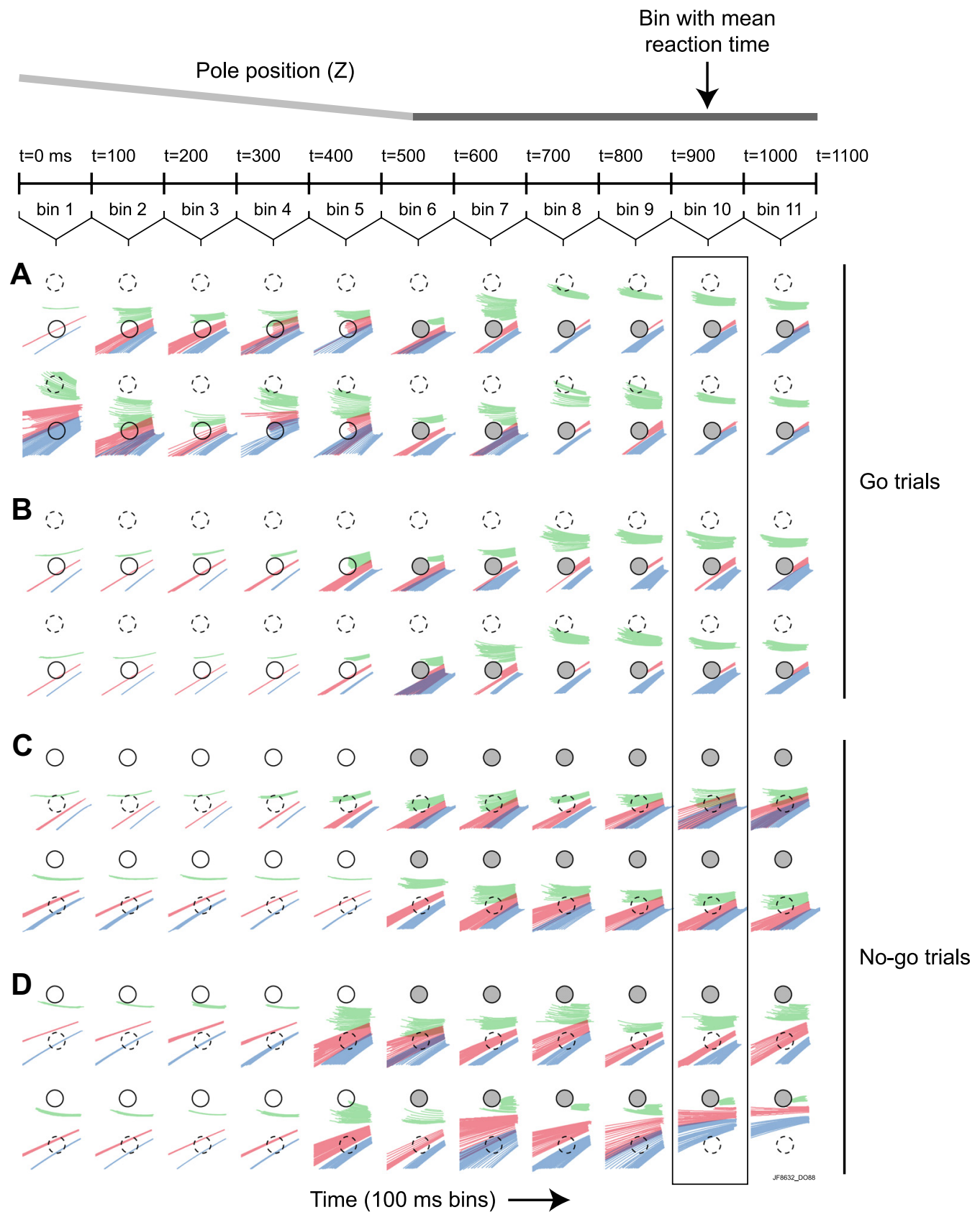

Figure 10. Whisking is directed and differs between go and no-go trials. Movie-style projections of three tracked whiskers (D4, green; $D 3$, red; $D 2$, blue) through time in consecutive $100 \mathrm{~ms}$ bins, for four go trials $(\boldsymbol{A}, \boldsymbol{B})$ and four no-go trials $(\boldsymbol{C}, \boldsymbol{D})$. Each row of projections depicts a single trial. Anterior is at top. Each $100 \mathrm{~ms}$ bin is the projection of whiskers through 50 frames (acquired at 500 $\mathrm{Hz}$ ) and shows the region of space explored within that $100 \mathrm{~ms}$ period. There are 11 bins covering the period from 0 to $1.1 \mathrm{~s}$, arranged left to right. The light gray slanted bar at top indicates approximate travel time of the pole on its descent. The dark gray horizontal bar indicates that the pole is in its bottom position. Trials are from a single behavioral session. The solid black circles depict the pole location. The dashed black circles indicate the position of the pole on the other type of trial. The gray fill in the circles indicates that the pole is at the bottom of its range and within reach of the whiskers in that time bin. The vertical black box indicates the bin containing the mean reaction time. $A$, Example ( $g$ ) trials in which the whiskers are in motion during the first couple hundred milliseconds of the trial, before the pole is in reach. $\boldsymbol{B}$, Trials in which the whiskers start moving immediately before the pole is accessible, or around the same time that the pole is accessible. $\boldsymbol{C}$, No-go trials in which the mouse searches the go position and avoids the no-go position, even though the pole is in the no-go position. $D$, No-go trials in which the mouse primarily searches the go position but also whisks forward into the no-go position. This type of trial is less common that the type shown in $C . \boldsymbol{A}-\boldsymbol{D}$, In many trials, the mouse has positioned at least one of its whiskers in the path of the go stimulus, in a position more protracted than the resting position of the whiskers. After initial contact with the pole on go trials, the mouse pressed D4 (green) against the pole for $>100 \mathrm{~ms}$ before protracting past the pole (toward the top of each image) to make a lick response.

Mice make absolute localizations to better than $6^{\circ}(<1 \mathrm{~mm})$ We next explored the limits of vibrissa-based object localization in mice. Psychometric curves relate performance to the size of the offset $(D)$ between go and no-go stimulus positions (Fig. 7). After training mice on an easy version of the task ( $D=4.29 \mathrm{~mm}$ offset $)$, we found that mice immediately performed harder localizations in which the no-go (distracter) stimulus had been moved closer to the go (target) stimulus. All mice performed above chance at $D=0.95 \mathrm{~mm}$, corresponding to $\sim 6^{\circ}$ of azimuthal angle (Fig. $7 A, B)$. Individual mice performed above chance level at $D=0.48$ $\mathrm{mm}$, or $\sim 2.8^{\circ}$ of azimuthal angle (Fig. $7 A$ ), although this effect was only marginally significant (one-sided binomial test, mouse JF3465, $p=0.0625$ ). Receiver-operating characteristic plots, in which hit rate is plotted against false alarm rate, show that de- 

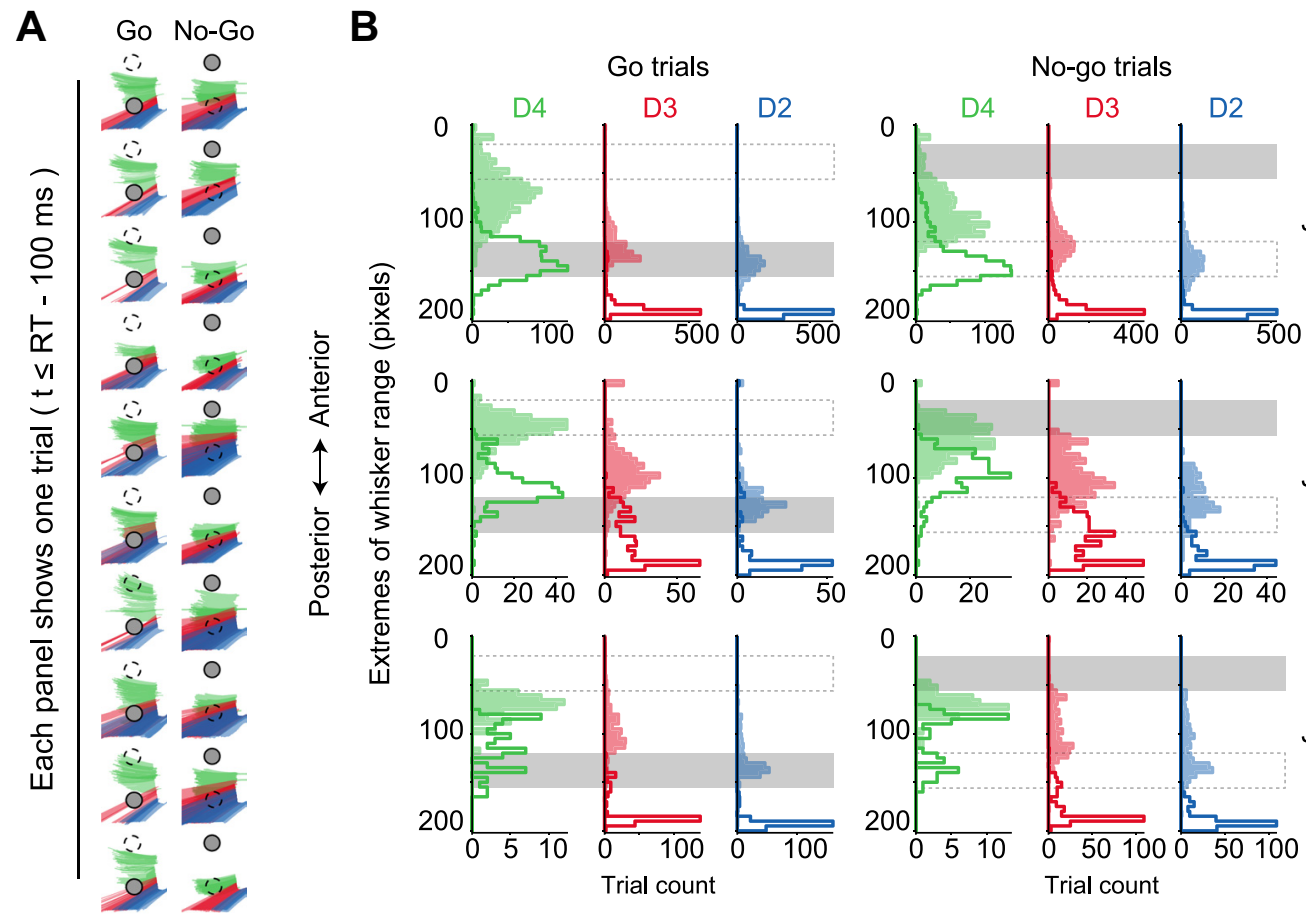

JF8632
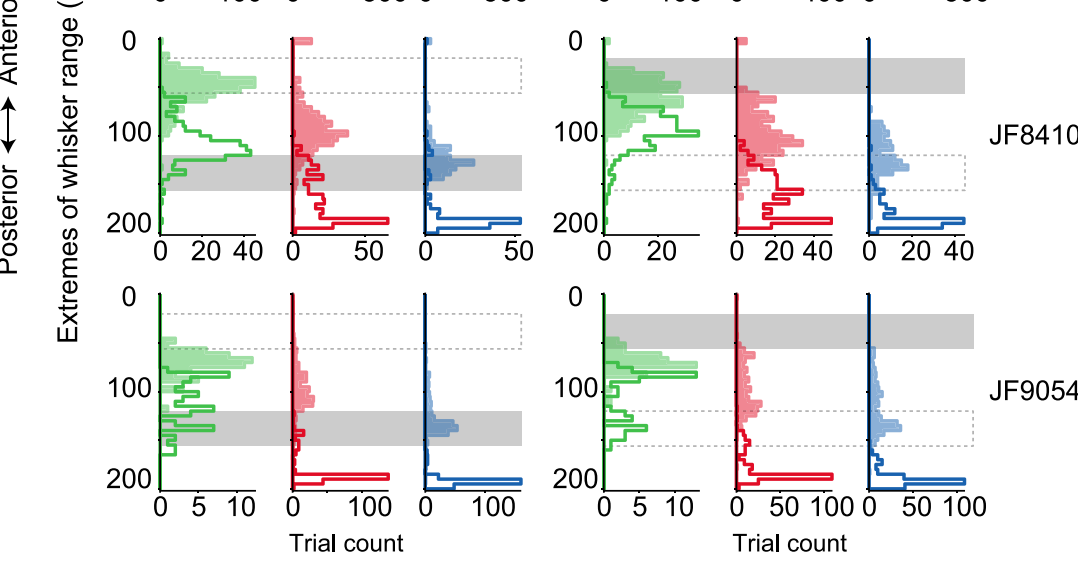

Figure 11. Mice predominantly explore the region of the rewarded (go) stimulus and avoid the no-go stimulus. $A$, Projections of three tracked whiskers (D4, green; D3, red; $D 2$, blue) through time for $10 \mathrm{go} \mathrm{trials} \mathrm{(left} \mathrm{column)} \mathrm{and} 10$ no-go trials (right column). Each rectangular projection shows an individual trial including all frames up to $100 \mathrm{~ms}$ before the mean reaction time (calculated across all tracked trials for the session) and provides a view of the space explored by the mouse before its reaction time. Anterior is at top. Trials are from a single behavioral session and are in order of consecutive presentation (although sorted into go and no-go), with trial number increasing from top to bottom. The solid black circles with gray fill depict the location of the pole. The dashed black circles indicate the (not-present) position of the pole for the other category of trials. Mice move their whiskers mainly through the region of the go stimulus and avoid the no-go stimulus position. B, Histograms show the anterior (filled) and posterior (open) extremes of whisker movement relative to the go and no-go stimulus positions, for each of three whiskers (D4, green; D3, red; D2, blue) and for three mice (JF8632, top row; JF8410, middle; JF9054, bottom). Whisker movement ranges for each trial were computed using all frames up to 100 ms before the mean reaction time. Trials are separated into go (left column) and no-go (right column). The anterior-posterior extent of the go and no-go stimuli are indicated by gray horizontal bars; the stimulus actually present for the given trial type (go or no-go) is shaded, whereas the other stimulus appears in dashed outline. Anterior is toward the top of each panel. Position was measured at the lateral distance of the medial edge of the poles. Although the whiskers often moved into the no-go stimulus position on no-go trials, the more common behavior was for the whiskers to search the go position and avoid the no-go position.

creases in performance at smaller offsets were attributable to both a decrease in hit rate and to an increase in false alarm rate, although the increased false alarm rate dominated the deterioration of performance (Fig. 7B). It is important to emphasize that mice made absolute or memory-guided localizations; thresholds for relative localizations in which both stimuli are simultaneously present are likely to be lower. In addition, prolonged training at smaller offsets would likely yield substantially lower behavioral thresholds.

\section{Tracking whisker position and deformation for thousands of trials}

We acquired high-speed (500 frames/s) video for $\sim 18,000$ behavioral trials. In most cases, each video sequence spanned $1.1 \mathrm{~s}$, starting at the beginning of the trial until after the mouse had responded; a subset of sequences $(\sim 500)$ spanned $2.106 \mathrm{~s}$ (Fig. $1 D$ ). Because the mice were head-fixed, we were able to record a stationary region of interest and still obtain high-resolution video of all the whiskers as they interacted with the pole (Fig. $8 A, B$ ) (see also Materials and Methods). In the majority of trials, whiskers had been trimmed to a single row and we were able to identify and track all individual whiskers throughout the video sequence. Tracking was performed using custom software (see Materials and Methods). Tracking converted the raw video into a subpixel resolution curve, corresponding to the medial axis of each whisker. We used this representation to compute the azimuthal angle near the base of the whisker $(\theta)$ (Fig. $8 \mathrm{C}$ ) and the position of the base. This represents the motor program underlying the object localization task. We also computed the change in whisker curvature $(\Delta \kappa)$ (Fig. $8 D$ ), which is proportional to the moment acting on the whisker (Neimark, 2001; Birdwell et al., 2007). This is one measure of the sensory input available to the animal. Axial forces, pushing the whisker into the follicle, which will also vary as the whisker bends against an object, are not presented here (Stüttgen et al., 2008). We tracked 3543 video sequences (each corresponding to a single trial), most including two to four whiskers, across a total of 2,054,280 video frames. This large number (far in excess of the tens of videos or fewer reported in previous studies [but see the study by Knutsen et al. (2006), who obtained 2357 tracked trials]) allowed us to obtain a statistical description of the object localization strategies used by mice in our task.

\section{Mice whisk in a directed and intelligent manner during object localization \\ Whisking can be bilaterally asymmetric and stereotyped}

Whisking during object localization differed from the exploratory whisking (Welker, 1964; Berg and Kleinfeld, 2003). Most conspicuously, whisking was typically bilaterally asymmetric (Fig. 9). Whisking usually started as the pole was descending before it was in reach of the whiskers (Fig. 9A). On the stimulus side, the whiskers moved toward (in this case, via a retractive movement) (for a description of stimulus positioning, see Materials and Methods and Table 1) the go-pole position, regardless of trial type, indicating that the mouse was searching for the 
go stimulus (Fig. 9A,C). The whiskers dwelled in this retracted position until the end of the trial. In contrast, the whiskers on the contralateral side moved rhythmically $(16.5 \mathrm{~Hz}$, mean across $N=3$ mice) around a set point somewhat protracted from baseline (Fig. 9A,C). This asymmetry was observed at different difficulty levels (offsets, $D$ ) (Fig. 9B,C).

Whisking is directed and differs between go and no-go trials

A slightly different pattern of whisking was seen when go and no-go pole positions were more anterior compared with those of Figure 9 (for a description of stimulus positioning, see Materials and Methods and Table 1). At the start of the trial, whiskers were usually held in a somewhat anterior, nonresting position, close to the go-pole position (Fig. 10; supplemental Fig. 5, available at www.jneurosci.org as supplemental material). In some cases, whisking started in the first few hundred milliseconds of the trial, well before the stimulus was within reach of the whiskers (Fig. $10 \mathrm{~A}$; supplemental Fig. 5, available at www.jneurosci.org as supplemental material). In other cases, whisking started immediately before (within $\sim 100 \mathrm{~ms}$ ) the pole came within reach of the whiskers, or even when the pole collided with a whisker in the path of the descending pole (Fig. $10 B$; supplemental Fig. 5, available at www.jneurosci.org as supplemental material). By the time the pole was in reach of the whiskers, the sequence of whisker movements became stereotyped (supple-

mental Fig. 5, available at www.jneurosci.org as supplemental material) and different between go and no-go trials (Fig. 10, compare $A, B$ with $C, D$; supplemental Fig. 5, available at www. jneurosci.org as supplemental material). Typically, in go trials an initial whisker-pole contact was followed by additional protraction or retraction against the pole (each mouse seemed to favor one whisker for this purpose) such that at least one whisker bent considerably, as if the mouse were confirming the presence of the pole in the go position (Fig. 10 A, B; supplemental Fig. 5, sixth and seventh time bins, available at www.jneurosci.org as supplemental material). Later in go trials, whisker D4 (green) protracted as the mouse moved to make a lick response (Fig. 10A, B; supplemental Fig. 5, last four time bins, available at www.jneurosci.org as supplemental material).

In no-go trials, the mouse continued to whisk, preferentially around the location of the go-pole position as if searching for it (Fig. 10C; supplemental Fig. 5, available at www.jneurosci.org as supplemental material), occasionally contacting the pole in the no-go position (Fig. 10D; supplemental Fig. 5, available at www. jneurosci.org as supplemental material). In a few cases, mice simply maintained a protracted position that put a whisker in the path of the go stimulus and did not whisk additionally if there was no contact with the whisker (indicating a no-go trial) (supplemental Fig. 5, available at www.jneurosci.org as supplemental material).
Mice tended to direct their whisking to the position of the go stimulus, avoiding the no-go-pole position (Fig. 11). There were plenty of exceptions, in which mice contacted the pole in the no-go position with their whiskers; indeed, all mice contacted the pole in the no-go position on some trials (Knutsen et al., 2006). But for easy trials $(D=4.29 \mathrm{~mm}$ offset $)$, the dominant motif was to contact the pole on go trials and to avoid the pole on no-go trials (Fig. 11 $A, B$ ). The mice therefore used a spatial sampling strategy in which contact between whisker and pole was primarily indicative of a go trial. That is, they appeared to convert a designed "discrimination" task into a "detection with distracters" task.

Whisker-pole contact per se, however, was not a sufficient cue for making a go response (Fig. 12). Mice correctly withheld lick responses on many no-go trials after contacting the pole in the no-go position (Fig. 12). In addition, the probability of at least one whisker-pole contact in the no-go position depended on the overall anterior-posterior positioning of the stimulus pair (Fig. 12 , compare $A, B)$. In mice with full whisker fields, moreover, whisker-pole contact occurred on virtually every trial (Fig. 12B). Even in these cases in which every trial involved whisker-object contact, mice still used the strategy of directing whisker movements to the location of the go stimulus such that the number of whisker-pole contacts was far higher on go trials (data not shown). 

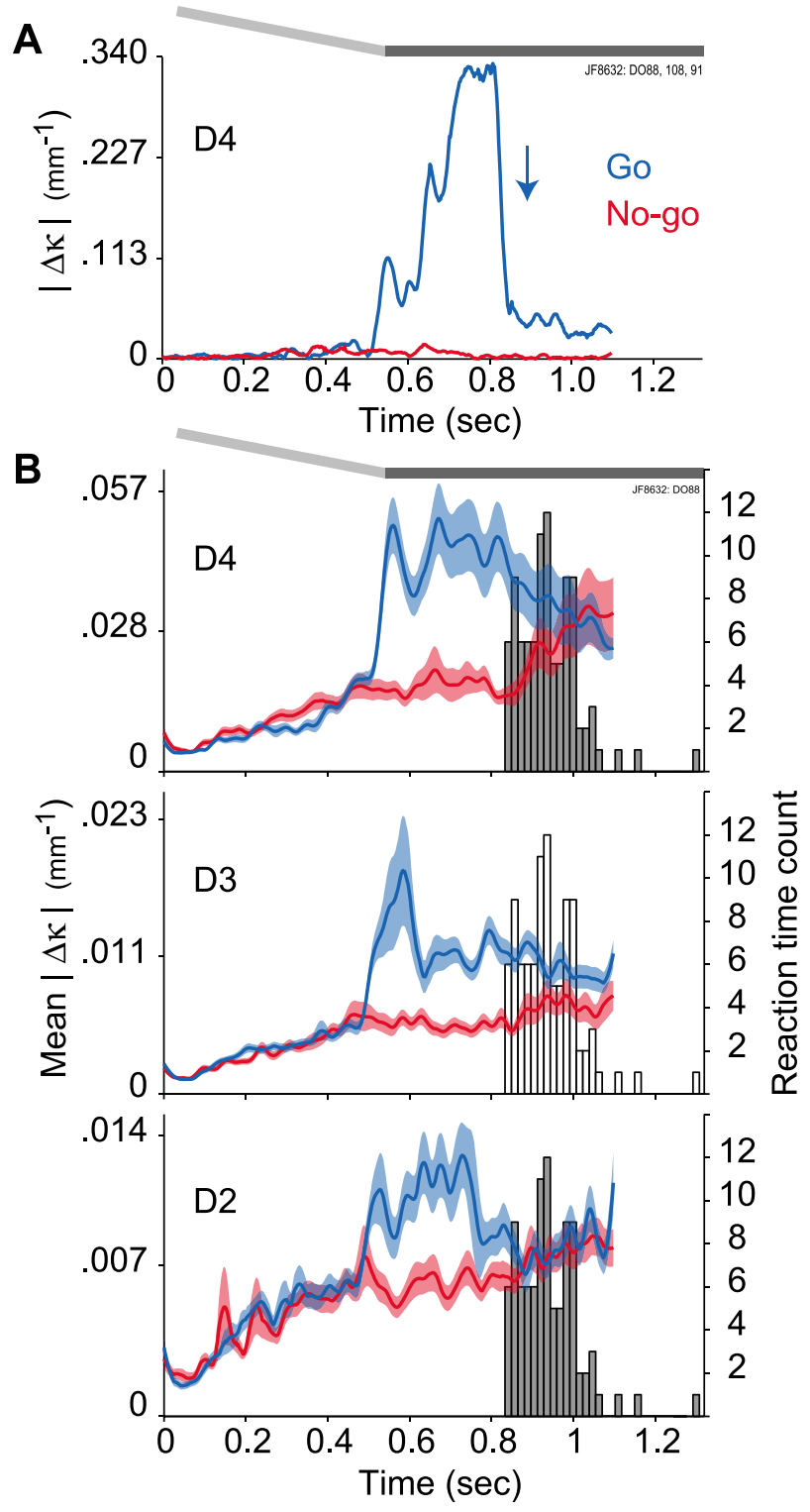

Figure 13. Whisker curvature changes diverge for go and no-go trials, well before the reaction time. $\boldsymbol{A}$, Rectified change in curvature for whisker D4 for a go (blue) and a no-go (red) trial. The blue arrow indicates the reaction time for the go trial. The light gray slanted bar at top indicates approximate travel time of the pole on its descent. The dark gray horizontal bar indicates that the pole is in its bottom position. Change in curvature $(\Delta \kappa)$ was computed as curvature minus the mean curvature in the first $100 \mathrm{~ms}$ of each trial. Traces were smoothed (before rectification) with a 50 ms Savitsky-Golay second-order filter. $\boldsymbol{B}$, Average rectified $\Delta \kappa$ for three whiskers (D4, D3, D2) across all go (blue) and no-go (red) trials for the behavioral session in $\boldsymbol{A}$ (error shading, \pm SEM). The distribution of reaction times for the go trials is shown in the middle panel and duplicated in gray in the top and bottom panels. The light gray slanted and dark gray horizontal bars are as in $\boldsymbol{A}$. Traces from each trial comprising the average were smoothed as in $\boldsymbol{A}$. The average traces were also smoothed with a 50 ms Savitsky-Golay filter. $N_{\text {go }}=95$ trials; $N_{\text {no-go }}=82$ trials.

\section{Mechanical forces diverge for go and no-go trials before the reaction time}

Mice appeared to maximize contact with the go (rewarded) stimulus and minimize contact with the no-go (unrewarded) stimulus. By using directed whisking, mice were thus able to amplify differences in mechanical input transduced at the whisker follicles. That is, the go and no-go stimuli differed not only in their positions (which they did by definition) but also in the probability and magnitude of forces based on contact between object and whisker. Mice did not simply make a light touch of the pole when it was in the go position. Instead, they pushed at least one whisker against the pole with enough force to cause significant bending (Figs. 8, 10; supplemental Fig. 5, available at www.jneurosci.org as supplemental material). In fact, changes from baseline whisker curvature were apparent and clearly diverged for go and no-go trials (Fig. 13). This separation of whisker curvature changes for the two trial types occurred on average 200-350 ms before the mouse's earliest reaction times (and 300-500 ms before the mean reaction time) (Fig. 13). Changes from baseline curvature are proportional to moment on the whisker (Birdwell et al., 2007); axial forces acting along the axis of the whisker will also change as whisker curvature changes after whisker-object contact. Because these highly different patterns of whisker curvature change would likely evoke different patterns of barrel cortex activity, they could provide a basis for the mouse's decision.

\section{Whisker kinematics during object localization}

In this section, we report measurements of whisker kinematics during the absolute object localization task (Figs. 14-20).

\section{Whisking speeds and angular ranges}

We measured whisker velocities and angular ranges across 3543 trials from six mice. Peak whisker velocities reached up to $\sim 10,000^{\circ}$ s (Fig. $14 A, C$ ). The highest velocities were attributable to stick-slip events in which the whisker caught on the pole and then abruptly slipped past it (supplemental Fig. 6, available at www.jneurosci.org as supplemental material). Average velocities were $\sim 10$-fold lower (Fig. $14 B, D$ ). This reflects the fact that whiskers spent a significant amount of time stationary, both before onset of the stimulus and after the mouse's decision to withhold a response on correct rejection trials (in contrast, on hit trials we found that licking at the lickport was invariably accompanied by whisking) (data not shown). There was a slight tendency for the highest absolute velocity on a trial to occur during retraction, as opposed to during protraction (median difference in peak absolute velocity during protraction vs during retraction, $-28 \pm 3 \%$ s; bootstrap SE; 9081 observations pooled across all whiskers and across three mice) (Carvell and Simons, 1990). Interestingly, peak velocity distributions differed among mice, with some mice consistently using faster whisker movements than others (Fig. 15) [two-tailed, two-sample Kolmogorov-Smirnov (K-S) tests, all 15 pairwise comparisons among six mice, $p<$ $0.001]$. There was no clear relationship between velocity and performance (data not shown).

The maximal angular range spanned by individual whiskers over the course of a trial ranged from $\sim 0$ to $100^{\circ}$ (Fig. 16). These angular ranges depended on the trial type (Fig. 16A) (two-tailed, two-sample K-S test on hits vs false alarms, data pooled across whiskers and $N=3$ mice, $p<0.001$ ), with false alarm trials greater than other types. Angular ranges depended also on the pole positions (Fig. 17), with more posterior pole positions (in which mice often made retractions toward the go position) producing a somewhat larger angular range (Fig. 17B) (correct rejections for $4.29 \mathrm{~mm}$ offset, pooled across stimulus-side whiskers; two-tailed, two-sample K-S test, $p<0.001)$. Individual differences among mice were also apparent in the angular ranges spanned by whiskers over the course of a trial (Fig. 18) (twotailed, two-sample K-S tests, 14 of 15 possible pairwise comparisons among six mice, $p<0.001$ ).

These results indicate that whisker movements are tailored to the demands of the task and depend on the stimulus configuration, the type of trial, and the individual mouse. The large ranges 
of whisker velocity $(\sim 0-10,000 \%$ s $)$ and angles $\left(\sim 0-100^{\circ}\right)$ indicate that headfixed object localization engages a large region of the space of possible whisker movements (Hill et al., 2008). The high peak whisker velocities seen in behaving mice underscore the need to explore these velocity regimes when performing experiments in anesthetized animals with experimenter-controlled whisker deflections (Ritt et al., 2008).

\section{Translational movement of whiskers is prominent}

Translation of the whiskers was a conspicuous feature of whisking in our headfixed mice (Fig. 19). In a projection of tracked whiskers across time, the apparent attachment point of the whisker (i.e., follicle position) moves along the face by up to several millimeters (Fig. 19A,B). In most trials, whisker angle and follicle position were highly correlated; whisker angle therefore predicted follicle position. For large-amplitude movements, follicles and whiskers moved in the same direction; for small-amplitude movements, the correlation was sometimes negative (Fig. $19 C-E)$. Follicle position and whisker angle are therefore under independent control, at least for small movements. Whisker translation may play a significant role in head-fixed object localization, underscoring the importance of monitoring the full whisker position (not just angle) using high-speed video (Harvey et al., 2001).

\section{Whisking patterns and reaction times depend on task difficulty}

While measuring psychophysical curves to obtain localization thresholds, we also acquired high-speed video from three mice while they solved localizations at three different levels of difficulty $(D=$ $4.29,2.38$, and $0.95 \mathrm{~mm}$ ). These videos allowed us to track the position of whiskers on both the stimulus side and the contralateral side. In addition, for these experiments we tracked jaw and tongue movement in the high-speed video, allowing us to measure reaction time as the latency from the start of the descent of the pole to the moment at which the mouse's tongue first emerged from its mouth. This yields a more precise reaction time than measurements at the lickport, because we observed that on occasion the mouse's first lick attempt did not interrupt the photobeam to record a response (data not shown). Reaction times for the hardest localizations $(D=0.95 \mathrm{~mm})$ were significantly longer than for easy localizations $(D=4.29 \mathrm{~mm})$ for two of the three mice (Fig. 20A) (one-sided, two-sample K-S test: mouse JF4004, $p<0.001$; mouse JF4793, $p=0.012$ ). The third mouse showed no significant difference in reaction times (Fig. 20A) $(p=0.1692)$. This mouse (JF3465) also had the worst performance at the $D=0.95$ mm localization (Fig. 7A, blue data points) (two-tailed $t$ tests against JF4793 and JF4004 row C-only condition, both $p<0.01$ ).

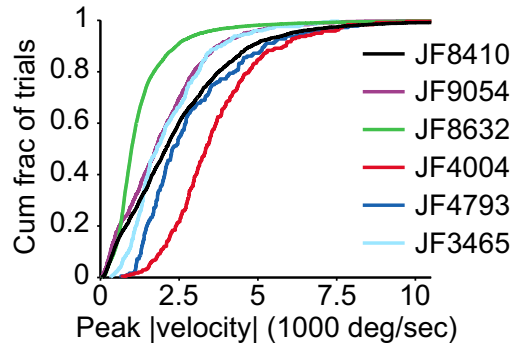

Figure 15. Different mice show different distributions of peak absolute velocity. Distributions of peak rectified velocity from each trial for six mice. Data are pooled across whiskers, trial types, stimulus and contralateral sides, and go/no-go position offsets.

Thus, in some but not all mice, reaction time is longer for more difficult localizations.

All mice, however, showed significant differences in whisking behavior for easy and difficult tasks (Fig. 20B-D). We tracked the 
A

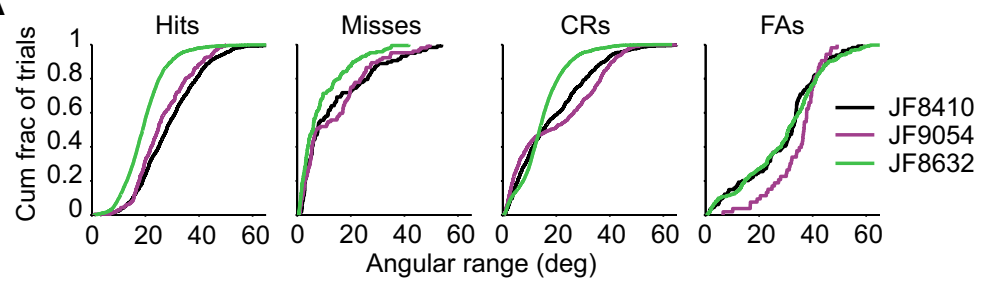

B

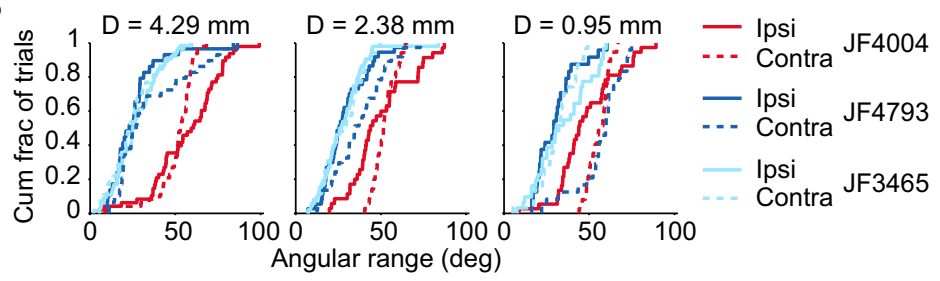

Figure 16. Whiskers explore an angular range between 0 and $100^{\circ}$ that depends on the trial type and the mouse. $A$, Maximum angular range traversed during a trial by each whisker, across all tracked whiskers for three mice. Separate histograms are shown for each trial category: hits, misses, correct rejections (CRs), and false alarms (FAs). B, Maximum angular range traversed in each trial for three mice performing localizations at three difficulty levels $(D=4.29,2.38$, and $0.95 \mathrm{~mm})$, for both stimulus-side (solid lines) and contralateral-side (dashed lines) whiskers. All trials are correct rejections. Separate groups of three mice are shown in $\boldsymbol{A}$ and $\boldsymbol{B}$. Anterior-posterior location of stimuli was different for these two groups of mice (see Materials and Methods and Table 1).

A

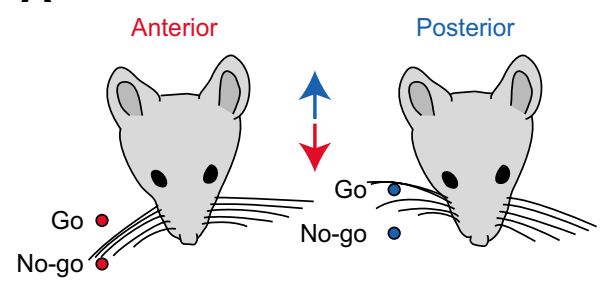

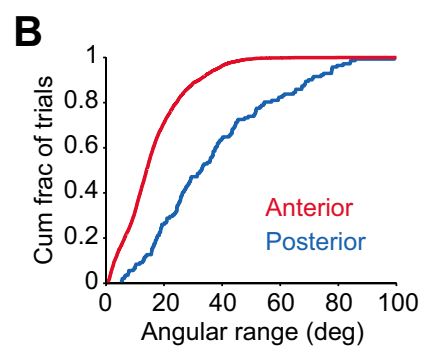

Figure 17. Maximal angular ranges explored during a trial depend on the positions of the pole stimuli. $\boldsymbol{A}$, Schematic of the two sets of pole positions used in the experiments reported here. The two pole positions (go and no-go) took either a more anterior (red) or a more posterior (blue) value, although the offset $(D)$ between the go and no-go pole positions was unchanged. $\boldsymbol{B}$, Distributions of maximum angular range traversed in each trial for the more anterior (protracted) and the more posterior (retracted) sets of positions. Mice explored a larger angular range when presented with the more posterior stimulus positions. Data are all from stimulus-side whiskers on correct rejection trials at the $D=4.29 \mathrm{~mm}$ offset and are pooled across whiskers.

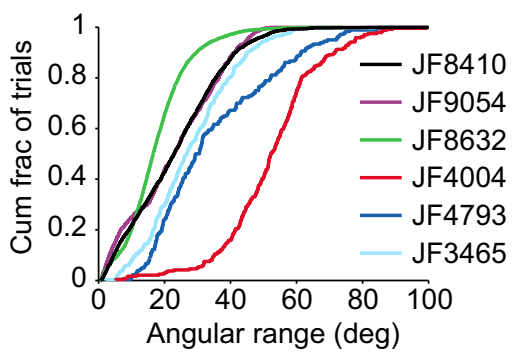

Figure 18. Different mice show different distributions of maximal angular range. Distributions of maximal angular range from each trial for six mice are shown. Data are pooled across whiskers, trial types, stimulus and contralateral sides, and go/no-go position offsets.

position of a single whisker on both the stimulus and the contralateral sides of each mouse on no-go trials. As a measure of overall whisking intensity (which can vary in frequency, amplitude, and duration), we calculated the mean absolute value whisker velocity over the entire video for each trial. Mean absolute velocity was significantly higher in the difficult $(D=0.95 \mathrm{~mm})$ compared with the easy $(D=4.29 \mathrm{~mm})$ localization for all three mice for the contralateral-side whiskers (two-tailed t tests, all $p<$
$0.001)$ and for one mouse on the stimulus side (Fig. $20 B)(p=0.021)$. For all mice, there was significantly less whisking on the stimulus side compared with the contralateral side (Fig. $20 \mathrm{~B}$ ) (two-tailed, twosample $t$ tests, all $p<0.001 ; N=3$ mice). However, inspection of the raw position data (for example, that shown in Fig. 9) revealed that mice made exquisitely precise movements of the whiskers into the position of the go stimulus, rather than whisking back and forth as on the contralateral side.

As a combined measure of whisking amplitude and stereotypy, we plotted mean change in whisker angle across all trials for the easy and hard localizations (Fig. 20C). This revealed systematic differences in the two conditions, with hard localizations showing a greater mean amplitude (Fig. 20C) (five of six comparisons, $p<0.03$; one-tailed bootstrap permutation test on difference in time window surrounding peak $[0.4,0.7 \mathrm{~s}]$ ) on both the stimulus and the contralateral sides.

We also computed the correlation coefficient between whisker angle time series for the stimulus and contralateral sides in the initial period (first $0.5 \mathrm{~s}$ ) of the trial, during which whisking typically began but before there was whisker-object contact (Fig. 20D). For two of the three mice, whiskers were more negatively correlated on difficult localizations compared with easy localizations (Fig. 20D) (two-sided, two-sample K-S tests, both $p<0.001$ ). The third mouse that did not show a significant difference (Fig. 20D) (mouse JF3465; $p=0.178$ ) was the same mouse that did not show a reaction time difference in Figure $20 \mathrm{~A}$.

For measurements reported in Figure 20, $A, C$, and $D$, data from the medium-difficulty localization $(D=2.38 \mathrm{~mm})$ were either intermediate between the easy $(4.29 \mathrm{~mm})$ and difficult (0.95 mm offset) localization data or not significantly different from at least one of the latter at our levels of statistical power. These medium-difficulty data are therefore omitted for clarity.

Overall, these results show clear differences between whisking strategies and between reaction times according to localization difficulty.

\section{Discussion}

We describe an object localization task for head-fixed mice. Mice learned the task within 1-2 weeks, performed at high levels ( $>90 \%$ correct), and could localize objects from memory to better than $0.95 \mathrm{~mm}\left(<6^{\circ}\right.$ of azimuth). The task depended on the barrel cortex and on contact between object and at least one whisker. Analysis of whisking revealed that mice searched in a strategic manner for the rewarded stimulus.

\section{A choice-based, active sensing task for head-fixed mice}

To our knowledge, this is the first report of head-fixed mice performing a choice-based task. Head-fixed rats have been used in tasks 
involving discrimination of whisker velocities (Stüttgen et al., 2006; Stüttgen and Schwarz, 2008) and for auditory discrimination (Otazu et al., 2009). Head fixation permits excellent stimulus control and behavioral monitoring and facilitates cellular neurophysiology (Crochet and Petersen, 2006; Dombeck et al., 2007). In mice, experiments with spontaneous (Crochet and Petersen, 2006; Dombeck et al., 2007; Ferezou et al., 2007; Poulet and Petersen, 2008) or reflexive (Boyden and Raymond, 2003) behaviors have begun to exploit experimental control afforded by head fixation.

Freely moving rodents can discriminate the roughness of textures (Guić-Robles et al., 1989; Carvell and Simons, 1990; von Heimendahl et al., 2007) and the widths of apertures (Krupa et al., 2001). Rodents also accurately judge the distances to platforms ("gap crossing") (Hutson and Masterton, 1986; Celikel and Sakmann, 2007) and the relative distance of two objects (Knutsen et al., 2006). Our paradigm is an adaptation of an absolute object localization task for partially restrained rats (Mehta et al., 2007). We find that head-fixed mice not only perform absolute object localization but do so at much higher levels of performance than partially restrained rats, perhaps because of the added positional certainty that comes with head fixation. Head-fixed mice allow unambiguous scoring of trials (Fig. 2) and will thereby permit powerful neurophysiological analyses and gain- and loss-offunction manipulations in a genetic model organism. Head fixation also has drawbacks. For example, it prevents the head movements seen during natural active sensation (Ritt et al., 2008).

\section{Absolute (memory-guided) localization thresholds \\ Mice performed absolute object localiza- tions to better than $0.95 \mathrm{~mm}$ in the anterior- posterior dimension ( $<6^{\circ}$ of azimuthal angle). Importantly, these localizations were memory-guided, in that only one of the two pos- sible stimuli was present at a time. This acuity is comparable with that of human observers localizing stimuli applied to the fingertips (Dallenbach, 1932; Loomis, 1979; Wheat et al., 1995). Studies with freely moving animals have shown that rats discriminate aperture widths that differ by $\sim 3 \mathrm{~mm}$ (Krupa et al., 2001), can discriminate the relative offset of two poles on either side of the head to $<1.5 \mathrm{~mm}$ (Knutsen et al., 2006), and can do absolute object localization to $15^{\circ}$ of azimuth (Mehta et al., 2007). Results from relative bilateral (Knutsen et al., 2006) and absolute (the present task) (Mehta et al., 2007) acuity tests cannot be directly compared, however, as absolute psycho- physical acuity is generally worse than relative acuity (Norrsell and Olausson, 1994; Recanzone et al., 1998).}

\section{Dependence of object localization on cortex}

Some whisker-dependent behaviors, including gap crossing (Hutson and Masterton, 1986), texture discrimination (Guić-
A
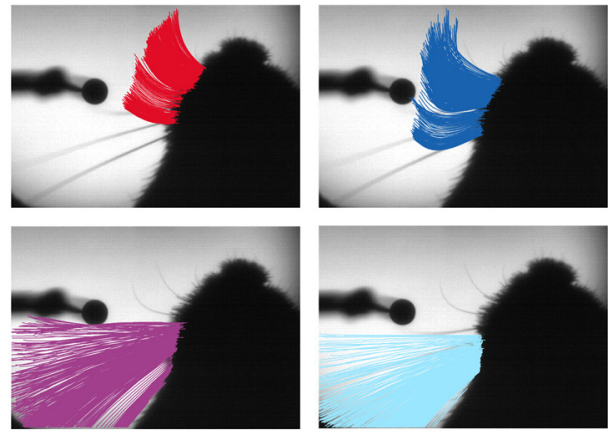

B
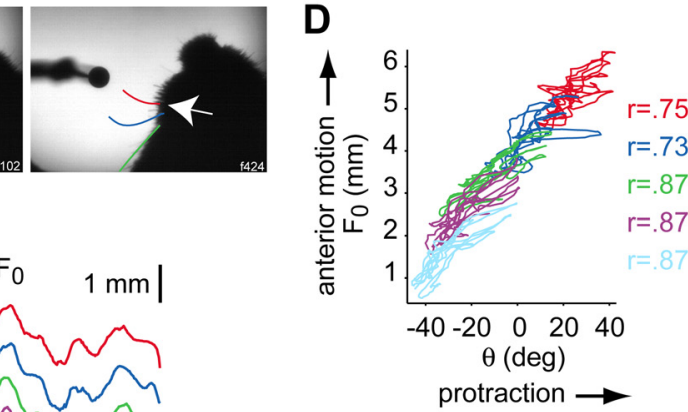

E

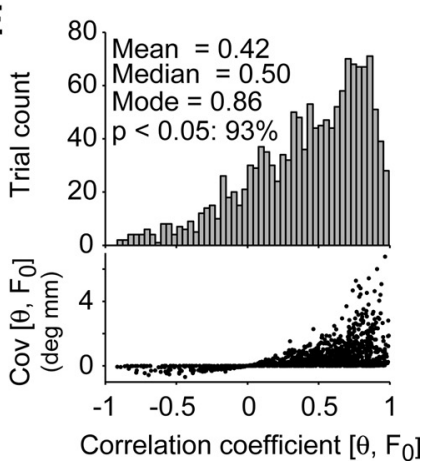

Figure 19. Translation of whiskers is prominent. $\boldsymbol{A}$, Projections of whiskers tracked through time for an example video highlight ranslation of the whisker base along the side of the snout. Whisker projections are superimposed on an arbitrary frame from the (top left frame) or separately for each whisker. $\boldsymbol{B}$, Pair of individua

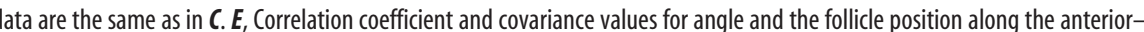
posterior axis for whisker D3 (see Materials and Methods). $N=1414$ correct rejection trials across three mice.

Robles et al., 1992), and aperture width discrimination (Krupa et al., 2001), appear to require the somatosensory cortex. In contrast, rats performing passive stimulus frequency discrimination are unaffected by lesions of the somatosensory cortex (Hutson and Masterton, 1986). Performance in the object localization task was completely abolished after silencing or lesions of the somatosensory cortex. These findings suggest that behaviors requiring active, as opposed to passive, vibrissa-based sensation require an intact barrel cortex.

\section{Head-fixed whisking}

Mice controlled the movement of their whiskers (but not their head) in solving the object localization task. We found that whisking was bilaterally asymmetric (Mitchinson et al., 2007; Towal and Hartmann, 2008) and that translational movement of the whisker pad was prominent (Harvey et al., 2001). Previous studies of head-fixed rodents have found the "exploratory" (Hill et al., 2008) and "discriminative" (Harvey et al., 2001) modes of 
A

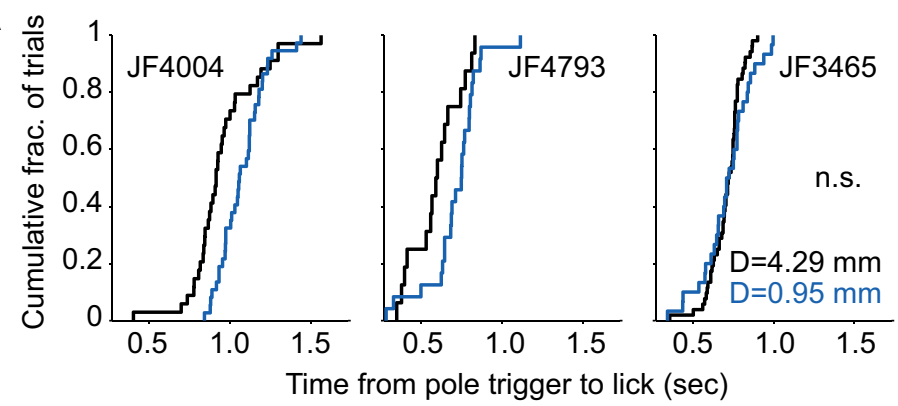

B

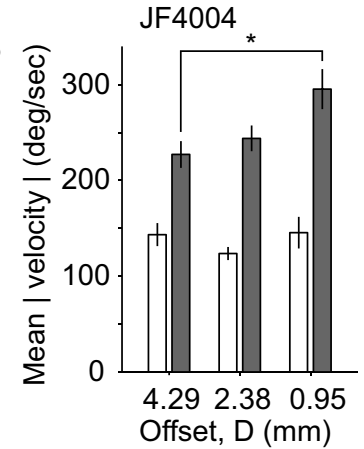

C

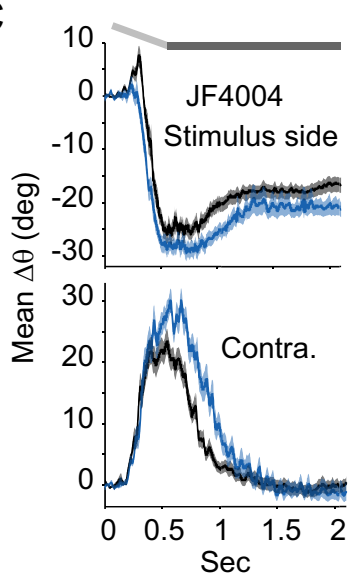

D

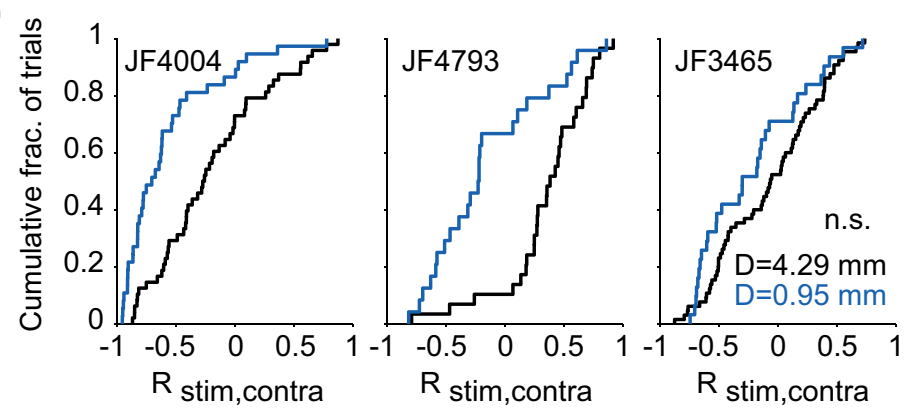

Figure 20. Whisking patterns and reaction times differ based on the required precision of localization but vary among mice. $\boldsymbol{A}$, Reaction times for lick responses measured directly from high-speed video on go trials. Reaction time is measured from the beginning of the pole descent until the mouse's tongue first leaves its mouth. Mouse JF4004 had row ( whiskers only. Mice JF4793 and JF3465 had full whisker fields. Two mice (JF4004 and JF4793) showed longer reaction times for the hard ( $D=0.95 \mathrm{~mm}$ offset) localizations than for the easy ( $D=4.29 \mathrm{~mm}$ ) localizations; reaction times for a third mouse (JF3465) were not statistically different. $\boldsymbol{B}$, Mean rectified whisker velocity, a measure of overall whisking intensity, revealed differences in whisking among localizations of different difficulty, for the contralateral whiskers in all three mice and for the stimulus-side whiskers for one mouse. In each case, increasing difficulty resulted in greater whisking intensity. For clarity, statistically significant differences are marked with asterisks only for comparisons between the $D=0.95 \mathrm{~mm}$ and $D=4.29 \mathrm{~mm}$ offsets. Error bars show bootstrap SEM. C, Average whisker movement amplitude is larger for difficult localizations, for whiskers on both the stimulus side (top row) and the contralateral side (bottom row). Mean change in whisker angle is a combined measure of amplitude and stereotypy of whisker movement. Change in whisker angle $(\Delta \theta)$ is computed as whisker angle minus the mean whisker angle in a $100 \mathrm{~ms}$ baseline period at the beginning of each trial. Error shading indicates \pm SEM. $D$, The correlation coefficient $\left(R_{\text {stim,contra }}\right)$ for whisker angle time

whisking to be highly similar between head-free and head-fixed conditions. We observed whiskers to reach peak velocities of up to $10,000^{\circ} / \mathrm{s}$, comparable with freely moving rats (Ritt et al., 2008). However, we cannot exclude the possibility that some features of whisker movements observed in head-fixed mice may reflect adaptation to head restraint.

We tracked whisker position and shape over time for thousands of trials, permitting a statistical description of the strategies used by mice to solve object localization. In addition, we report quantitative measurements of whisker deformation in behaving animals; such measurements are key to reconstructing forces on the follicle (Birdwell et al., 2007). Linking neuronal activity to sensory stimulation in the whisker-tobarrel pathway, and whisker movement to activity in motor pathways, will require reconstruction of both whisker kinematics and dynamics across many trials. We have shown here that this is feasible in head-fixed mice performing object localizations.

\section{Intelligent and directed whisking strategies for object localization}

By analyzing whisker position as a function of time, we reconstructed the motor strategy of the mice. Measuring the shape of the whisker provided information about the mechanical inputs to the whisker system. Mice solved the object localization task with a directed and intelligent whisking scheme.

All mice used a similar strategy in solving the object localization task. Mice tended to preferentially explore the region of, and make contact with, the rewarded (go) stimulus and made far fewer contacts with the unrewarded (no-go) stimulus. Two previous studies in freely moving (Knutsen et al., 2006) and partially restrained (Mehta et al., 2007) rats conclude that rodents perform relatively symmetrical whisking with respect to anterior and posterior object locations. In the study by Knutsen et al. (2006), rats had to determine whether a pole was posterior or anterior to a comparison pole presented to the other side of the head. Rats frequently touched the pole in its more anterior po-

\section{$\leftarrow$}

series between a whisker on the stimulus side and a whisker on the contralateral side, computed in the first $0.5 \mathrm{~s}$ of the trial (see Results). Whisker movements are more negatively correlated on difficult compared with easy localizations for two of three mice. $\boldsymbol{A}-\boldsymbol{D}$, Same data set appears in part in Figure 9. 
sition, after whisking through and not finding the pole in the more posterior position. They argue that rats explored both the go and no-go positions. However, a substantial fraction of animals did not routinely scan the full distance to the anterior pole. Furthermore, it is possible that rats made their somatosensory decision before contacting the anterior pole.

Mehta et al. (2007) analyzed object localization in partially restrained rats and found relatively symmetrical whisking with respect to go and no-go positions, unlike our mice. However, some rats clearly showed asymmetric whisking [Mehta et al. (2007), their Fig. 6C,F]. In addition, the task performance of the rats was relatively poor, with a high incidence of false alarms. It is likely that whisking strategies change with training and might differ between inexperienced and expert animals.

Whisking strategies will also vary with the details of the task. For example, we have trained head-fixed mice to discriminate pole position along the radial direction (along the whisker), so that the poles at go and no-go positions are contacted at identical azimuthal angles (Pammer et al., 2009). Under these conditions, mice must adopt an alternative strategy for object localization.

Mice often pressed whiskers against the stimulus pole and caused significant bending. This contrasts with freely exploring rats, which appear to minimize the bending of whiskers that contact obstructing objects (Mitchinson et al., 2007). Thus, depending on the task at hand, rodents regulate the magnitude of the forces at the follicle by pressing their whiskers against objects to a varying degree. More generally, the whisker movements required to solve specific whisker-based localization tasks differ: in aperture width discrimination whisking is not required (Krupa et al., 2001), whereas whisking is required to determine the relative distance of two objects (Knutsen et al., 2006).

Individual mice showed quantitatively different profiles of whisking behavior, especially in terms of velocity distributions, angular range distributions, and differences in whisking associated with solving localizations of varying difficulty. Individual differences associated with task performance and strategy have also been observed during active somatosensation in both humans (Gamzu and Ahissar, 2001) and rats solving tactile discriminations (Carvell and Simons, 1995).

What does the strategy used by mice to solve our object localization task imply for sensory coding? Neurons in the whiskerto-barrel pathway respond phasically to passive (Simons, 1978; Brecht and Sakmann, 2002) and active (Szwed et al., 2006; Yu et al., 2006) contact with objects. By maximizing whisker contact with the go stimulus, and minimizing contact with the no-go stimulus, mice used a motor strategy that likely produces highly different patterns of activity across barrel cortex for go and no-go trials and that might therefore help the mouse choose between go and no-go responses.

\section{Summary}

Here, we have described an absolute object localization task for head-fixed mice that depends on the whiskers and on somatosensory cortex. Mice performed localizations using a sophisticated strategy expected to yield large differences in barrel cortex activity patterns for different trial types. This and related behavioral paradigms for head-fixed mice, together with new tools for monitoring and manipulating activity in specific neural circuits, promise to illuminate the neural basis of perceptual decision making.

\section{References}

Berg RW, Kleinfeld D (2003) Rhythmic whisking by rat: retraction as well as protraction of the vibrissae is under active muscular control. J Neurophysiol 89:104-117.
Birdwell JA, Solomon JH, Thajchayapong M, Taylor MA, Cheely M, Towal RB, Conradt J, Hartmann MJ (2007) Biomechanical models for radial distance determination by the rat vibrissal system. J Neurophysiol 98:2439-2455.

Boyden ES, Raymond JL (2003) Active reversal of motor memories reveals rules governing memory encoding. Neuron 39:1031-1042.

Brecht M, Sakmann B (2002) Dynamic representation of whisker deflection by synaptic potentials in spiny stellate and pyramidal cells in the barrels and septa of layer 4 rat somatosensory cortex. J Physiol 543:49-70.

Carvell GE, Simons DJ (1990) Biometric analysis of vibrissal tactile discrimination in the rat. J Neurosci 10:2638-2648.

Carvell GE, Simons DJ (1995) Task- and subject-related differences in sensorimotor behavior during active touch. Somatosens Mot Res 12:1-9.

Celikel T, Sakmann B (2007) Sensory integration across space and in time for decision making in the somatosensory system of rodents. Proc Natl Acad Sci U S A 104:1395-1400.

Crochet S, Petersen CC (2006) Correlating whisker behavior with membrane potential in barrel cortex of awake mice. Nat Neurosci 9:608-610.

Dallenbach KM (1932) A comparative study of the errors of localization on the finger-tips. Am J Psychol 44:327-331.

Diamond ME, von Heimendahl M, Knutsen PM, Kleinfeld D, Ahissar E (2008) "Where" and "what" in the whisker sensorimotor system. Nat Rev Neurosci 9:601-612.

Dombeck DA, Khabbaz AN, Collman F, Adelman TL, Tank DW (2007) Imaging large-scale neural activity with cellular resolution in awake, mobile mice. Neuron 56:43-57.

Dörfl J (1982) The musculature of the mystacial vibrissae of the white mouse. J Anat 135:147-154.

Evarts EV (1968) Relation of pyramidal tract activity to force exerted during voluntary movement. J Neurophysiol 31:14-27.

Ferezou I, Haiss F, Gentet LJ, Aronoff R, Weber B, Petersen CC (2007) Spatiotemporal dynamics of cortical sensorimotor integration in behaving mice. Neuron 56:907-923.

Gamzu E, Ahissar E (2001) Importance of temporal cues for tactile spatialfrequency discrimination. J Neurosci 21:7416-7427.

Guić-Robles E, Valdivieso C, Guajardo G (1989) Rats can learn a roughness discrimination using only their vibrissal system. Behav Brain Res 31:285-289.

Guić-Robles E, Jenkins WM, Bravo H (1992) Vibrissal roughness discrimination is barrelcortex-dependent. Behav Brain Res 48:145-152.

Harvey MA, Bermejo R, Zeigler HP (2001) Discriminative whisking in the head-fixed rat: optoelectronic monitoring during tactile detection and discrimination tasks. Somatosens Mot Res 18:211-222.

Hill DN, Bermejo R, Zeigler HP, Kleinfeld D (2008) Biomechanics of the vibrissa motor plant in rat: rhythmic whisking consists of triphasic neuromuscular activity. J Neurosci 28:3438-3455.

Hutson KA, Masterton RB (1986) The sensory contribution of a single vibrissa's cortical barrel. J Neurophysiol 56:1196-1223.

Knutsen PM, Derdikman D, Ahissar E (2005) Tracking whisker and head movements in unrestrained behaving rodents. J Neurophysiol 93:2294-2301.

Knutsen PM, Pietr M, Ahissar E (2006) Haptic object localization in the vibrissal system: behavior and performance. J Neurosci 26:8451-8464.

Krupa DJ, Matell MS, Brisben AJ, Oliveira LM, Nicolelis MA (2001) Behavioral properties of the trigeminal somatosensory system in rats performing whisker-dependent tactile discriminations. J Neurosci 21:5752-5763.

Loomis JM (1979) An investigation of tactile hyperacuity. Sens Processes 3:289-302.

Luo L, Callaway EM, Svoboda K (2008) Genetic dissection of neural circuits. Neuron 57:634-660.

Mehta SB, Whitmer D, Figueroa R, Williams BA, Kleinfeld D (2007) Active spatial perception in the vibrissa scanning sensorimotor system. PLoS Biol 5:e15.

Mitchinson B, Martin CJ, Grant RA, Prescott TJ (2007) Feedback control in active sensing: rat exploratory whisking is modulated by environmental contact. Proc Biol Sci 274:1035-1041.

Neimark MA (2001) The mechanics of whisking: the first stage in the transduction of surface textures into neural signals. In: Thesis No. 14527, Princeton University.

Norrsell U, Olausson H (1994) Spatial cues serving the tactile directional sensibility of the human forearm. J Physiol 478:533-540. 
O'Connor DH, Huber D, Svoboda K (2009) Reverse engineering the mouse brain. Nature 461:923-929.

Otazu GH, Tai LH, Yang Y, Zador AM (2009) Engaging in an auditory task suppresses responses in auditory cortex. Nat Neurosci 12:646-654.

Pammer L, O’Connor DH, Clack NG, Huber D, Komiyama T, Myers EW, Svoboda K (2009) Mice can discriminate tactile stimuli along their whisker (radial object distance). Soc Neurosci Abstr 35:174.5.

Poulet JF, Petersen CC (2008) Internal brain state regulates membrane potential synchrony in barrel cortex of behaving mice. Nature 454:881-885.

Recanzone GH, Makhamra SD, Guard DC (1998) Comparison of relative and absolute sound localization ability in humans. J Acoust Soc Am 103:1085-1097.

Ritt JT, Andermann ML, Moore CI (2008) Embodied information processing: vibrissa mechanics and texture features shape micromotions in actively sensing rats. Neuron 57:599-613.

Simons DJ (1978) Response properties of vibrissa units in rat SI somatosensory neocortex. J Neurophysiol 41:798-820.

Stüttgen MC, Schwarz C (2008) Psychophysical and neurometric detection performance under stimulus uncertainty. Nat Neurosci 11:1091-1099.

Stüttgen MC, Rüter J, Schwarz C (2006) Two psychophysical channels of whisker deflection in rats align with two neuronal classes of primary afferents. J Neurosci 26:7933-7941.

Stüttgen MC, Kullmann S, Schwarz C (2008) Responses of rat trigeminal ganglion neurons to longitudinal whisker stimulation. J Neurophysiol 100:1879-1884.

Szwed M, Bagdasarian K, Blumenfeld B, Barak O, Derdikman D, Ahissar E (2006) Responses of trigeminal ganglion neurons to the radial distance of contact during active vibrissal touch. J Neurophysiol 95:791-802.

Torre V, Poggio T (1986) On edge detection. MIT AI Memo 768.

Towal RB, Hartmann MJ (2008) Variability in velocity profiles during free air whisking behavior of unrestrained rats. J Neurophysiol 100:740-752.

Voigts J, Sakmann B, Celikel T (2008) Unsupervised whisker tracking in unrestrained behaving animals. J Neurophysiol 100:504-515.

von Heimendahl M, Itskov PM, Arabzadeh E, Diamond ME (2007) Neuronal activity in rat barrel cortex underlying texture discrimination. PLoS Biol 5:e305.

Welker WI (1964) Analysis of sniffing of the albino rat. Behavior 22:223-244.

Wheat HE, Goodwin AW, Browning AS (1995) Tactile resolution: peripheral neural mechanisms underlying the human capacity to determine positions of objects contacting the fingerpad. J Neurosci 15:5582-5595.

Wurtz RH (1968) Visual cortex neurons: response to stimuli during rapid eye movements. Science 162:1148-1150.

Yu C, Derdikman D, Haidarliu S, Ahissar E (2006) Parallel thalamic pathways for whisking and touch signals in the rat. PLoS Biol 4:e124. 